\title{
The current status of fine-tuning in supersymmetry
}

\author{
Melissa van Beekveld, ${ }^{a, b}$ Sascha Caron ${ }^{a, b}$ and Roberto Ruiz de Austri ${ }^{c}$ \\ ${ }^{a}$ Theoretical High Energy Physics, Radboud University Nijmegen, \\ Heyendaalseweg 135, 6525 AJ Nijmegen, the Netherlands \\ ${ }^{b}$ Nikhef, \\ Science Park 105, 1098 XG Amsterdam, the Netherlands \\ ${ }^{c}$ Instituto de Física Corpuscular, IFIC-UV/CSIC, \\ Valencia, Spain \\ E-mail: mcbeekveld@gmail.com, scaron@nikhef.nl, rruiz@ific.uv.es
}

ABSTRACT: In this paper, we minimize and compare two different fine-tuning measures in four high-scale supersymmetric models that are embedded in the MSSM. In addition, we determine the impact of current and future dark matter direct detection and collider experiments on the fine-tuning. We then compare the low-scale electroweak measure with the high-scale Barbieri-Giudice measure. We find that they reduce to the same value when the higgsino parameter drives the degree of fine-tuning. We also find spectra where the high-scale measure turns out to be lower than the low-scale measure. Depending on the high-scale model and fine-tuning definition, we find a minimal fine-tuning of 3-38 (corresponding to $\mathcal{O}(10-1) \%$ ) for the low-scale measure, and 63-571 (corresponding to $\mathcal{O}(1-0.1) \%)$ for the high-scale measure. We stress that it is too early to conclude on the fate of supersymmetry, based only on the fine-tuning paradigm.

KEYwORDS: Supersymmetry Phenomenology

ARXiv EPrint: 1906.10706 


\section{Contents}

1 Introduction 1

2 Supersymmetric models and their fine-tunings 3

2.1 SUSY models 4

2.2 The electroweak fine-tuning measure 5

2.3 The Barbieri-Giudice measure 5

2.3.1 Dependence on high-scale model assumptions 6

2.4 Concluding remarks 8

3 Analysis setup $\quad 8$

3.1 Implementation of the limits imposed by future experiments $\quad 10$

4 Results 11

4.1 mSUGRA 11

$\begin{array}{lll}4.2 & \text { mSUGRA-var } & 15\end{array}$

$\begin{array}{lll}4.3 & \text { NUHGM } & 18\end{array}$

$\begin{array}{ll}4.4 \text { pMSSM-GUT } & 21\end{array}$

$\begin{array}{llr}5 & \text { Conclusion } & 27\end{array}$

\section{Introduction}

The instability of the mass of a scalar particle under quantum corrections was firstly discussed in the pioneering work of Susskind [1], Veltman [2] and 't Hooft [3]. They showed that the existence of both a scalar particle, like the Higgs boson, and a much heavier particle that couples to this scalar particle implies that the mass of the scalar particle will receive radiative corrections that are quadratic in the mass of the heavy particle. They pointed out that if the Higgs boson indeed has a mass around the weak scale, and if the Standard Model (SM) is to be embedded in a high-scale (HS) theory, this HS theory would generally require extreme forms of fine-tuning (FT) to prevent the Higgs mass from becoming of the same order as the HS. This would result in an unnatural theory unless one constructs the HS theory in such a way that it is free from FT. Many years later, the Higgs boson with a mass of $125 \mathrm{GeV}$ has been found $[4,5]$, a value that is certainly not far from the weak scale. The existence of a HS theory could, therefore, introduce a FT problem.

There are many reasons to believe in a theory that complements the Standard Model (SM) at higher mass scales: there is no explanation/solution for the origin of neutrino masses or the hierarchies among fermion masses, the nature of dark matter, the origin of electroweak symmetry breaking (EWSB) or the possible instability of the Higgs vacuum. 
In addition, as gravity becomes strongly coupled around the Planck scale, the SM needs to be complemented by a theory of quantum gravity at this high scale. And beyond the Planck scale, at energies around $\mathcal{O}\left(10^{40}\right) \mathrm{GeV}$, we encounter the Landau Pole of U(1), signaling that the perturbative nature of the SM is bound to break down eventually. If the HS theory is to solve some or all of the aforementioned problems, it is likely that it somehow couples to the SM particles. As mentioned before, this HS theory would then have to be constructed in a careful way in order to avoid the Higgs mass FT problem.

The FT problem is closely related to how we perform physics and set up experiments: we always assume that physics on large length scales is not affected by physics on small length scales. For example, we do not expect that gravity influences the physics that we are probing at colliders and consequently do not take its effects into account when making predictions for the LHC. Therefore, the apparent break down of this assumption in the Higgs sector does require an explanation, as it is the only example we know of where low-scale physics is extremely sensitive to high-scale physics.

Supersymmetry (SUSY) is a theory that is able to solve the Higgs FT problem by imposing a space-time symmetry between bosons and fermions [6-8]. Realized in its minimal form, SUSY can explain the observed Higgs mass, predict EWSB from SUSY breaking and allow for a dark matter candidate if R-parity conservation is assumed. In addition, any (softly broken) supersymmetric extension of the SM ensures that quadratic quantum corrections to masses of scalar particles are absent, which remedies the FT problem.

The FT problem in SUSY is well-studied [9-41]. Since many years it is claimed that in order to have a natural version of the minimal supersymmetric extension of the SM (the MSSM), one expects SUSY particles with masses that lie well below the TeV scale [42-55]. However, to date, there has not been any evidence for the existence of SUSY particles at these scales. This has motivated some to go beyond the MSSM (see e.g. refs. [56-74]).

In this paper, we reanalyze the question: does the non-observation of SUSY particles make any minimal realization of a supersymmetric SM necessarily an unnatural theory? It is well known that the answer to this question is both a model dependent and measure dependent one $[21,75,76]$. It is then interesting to see how different measures and models compare, like was studied before in refs. [21, 75]. It is our aim to update and extend this work by including all current constraints on the MSSM parameter space. This will also allow us to examine to what extend the experiments have constrained this parameter space, and which experiment had the biggest impact on it. A natural follow up question, which is also one we will address here, is: what sort of experiment is needed to exclude the natural MSSM?

To answer both questions, one needs a measure of FT to quantify the degree of naturalness. We will explore two widely used FT measures: the electroweak (EW) measure [75] and the Barbieri-Giudice (BG) measure [14, 42], whose definitions will be given in section 2. Low values of these FT measures mean low degrees of fine-tuning: an FT value of 100 corresponds to $\mathcal{O}(1 \%)$ fine-tuning. ${ }^{1}$ Note that 'how much' FT one allows in the theory

\footnotetext{
${ }^{1}$ We note in passing that the inverse of the FT measure can also be interpreted either as a p-value [27] or in a Bayesian context [19, 77] to get the correct value of the Higgs boson mass.
} 
depends on the level of cancellation between different theory parameters one is willing to accept. There is no general consensus on this, which brings a certain degree of subjectivity to the discussion. But even if one would agree on an absolute maximum amount of FT that one is willing to tolerate, there is, in fact, a second complication. The sort of experiment that will exclude a natural version of the MSSM is, as mentioned above, a measure dependent one, which we will clarify in section 2 .

To access the impact of worldwide data on the FT, we will minimize, like was done for the first time in ref. [32], the FT for both measures in four different HS SUSY models (described in section 2). All of these HS models are embedded in the low-scale (LS) phenomenological Minimal Supersymmetric Standard Model (pMSSM) [78]. We then show how past and current experiments have constrained the FT. In addition, we study the ability of future ton-scale dark matter direct detection experiments and future colliders to constrain the FT in the four HS realizations. The set-up for this analysis can be found in section 3 and the results are reported in section 4 . We conclude in section 5 , where one may also find a comparison of our work with claims that previously have been made in the literature.

\section{Supersymmetric models and their fine-tunings}

Whether SUSY is realized in nature is unknown and as such, we do not know which and how many fundamental parameters exist for the actual HS theory. A generic broken SUSY theory has two relevant energy scales: a HS one $\left(M_{\mathrm{GUT}}\right)$ at which SUSY breaking takes place, and a LS one ( $M_{\text {SUSY }}$ ) where the resulting SUSY particle spectrum is situated and the EWSB conditions must be satisfied. The breaking conditions link the $Z$-boson mass $\left(M_{Z}\right)$ to the input parameters via the minimization of the one-loop scalar potential of the Higgs fields. The resulting equation is [13, 79]:

$$
\frac{M_{Z}^{2}}{2}=\frac{m_{H_{d}}^{2}+\Sigma_{d}^{d}-\left(m_{H_{u}}^{2}+\Sigma_{u}^{u}\right) \tan ^{2} \beta}{\tan ^{2} \beta-1}-\mu^{2},
$$

where $m_{H_{u}}$ and $m_{H_{d}}$ are the soft SUSY breaking Higgs masses, $\mu$ is the SUSY version of the SM Higgs mass parameter and $\tan \beta$ is the ratio of the vacuum expectation values of the two Higgs doublets. The two effective potential terms $\Sigma_{u}^{u}$ and $\Sigma_{d}^{d}$ denote the one-loop corrections to the soft SUSY breaking Higgs masses (explicit expressions are shown in the appendix of ref. [13]). All terms in eq. (2.1) are evaluated at $M_{\text {SUSY }}$.

In order to obtain the observed value of $M_{Z}=91.2 \mathrm{GeV}$, one needs some degree of cancellation between the SUSY parameters appearing in eq. (2.1). In the general case: if the needed cancellation is large, small changes in the SUSY parameters will result in a widely different value of $M_{Z}$, in which case the considered spectrum is fine-tuned. FT measures aim to quantify the sensitivity of $M_{Z}$ to the SUSY input parameters. In the literature one can find two main classes of SUSY FT measures: one that does take underlying model assumptions into account, such as the BG measure [14, 42], and one that does not, such as the EW measure [75]. To assess the differences between these two measures, we will look at four different HS SUSY models that can all be embedded in the (LS) pMSSM. After defining these HS models we will review the two FT measures in more detail. 


\subsection{SUSY models}

All of the HS SUSY models we will consider in this paper are embedded in the pMSSM [78], which is constructed as follows:

- The first and second generation squark and slepton masses are degenerate.

- All trilinear couplings of the first and second generation sfermions are set to zero.

- There are no new sources of CP violation.

- All sfermion mass matrices are assumed to be diagonal to ensure minimal flavor violation.

After applying these conditions one ends up with a 19-dimensional model that can be parametrized as follows: the sfermion soft-masses are described by the first and second generation squark masses $m_{\tilde{Q}_{1}}, m_{\tilde{u}_{R}}$ and $m_{\tilde{d}_{R}}$, the third generation squark masses $m_{\tilde{Q}_{3}}$, $m_{\tilde{t}_{R}}$ and $m_{\tilde{b}_{R}}$, the first and second generation of slepton masses $m_{\tilde{L}_{1}}, m_{\tilde{e}_{R}}$, and the third generation of slepton masses $m_{\tilde{L}_{3}}, m_{\tilde{\tau}_{R}}$. Only the trilinear couplings of the third generation of sfermions $A_{\tilde{t}}, A_{\tilde{b}}$ and $A_{\tilde{\tau}}$ are assumed to be non-zero. The Higgs sector is described by the ratio of the Higgs vacuum expectation values $\tan \beta$ and the soft Higgs masses $m_{H_{u, d}}$. Instead of these Higgs masses, it is custom to use the higgsino mass parameter $\mu$ and the mass of the pseudoscalar Higgs $m_{A}$ as free (input) parameters, which set the values for the soft Higgs masses via the requirement of EWSB (eq. (2.1)). Finally, the gaugino sector is described by $M_{1}, M_{2}$ and $M_{3}$. All of these parameters are defined at the scale $M_{\mathrm{SUSY}}$, which is taken to be the geometric average of the two stop pole masses $\left(\sqrt{m_{\tilde{t}_{1}} m_{\tilde{t}_{2}}}\right)$.

The HS models that we will consider are:

- $m S U G R A$ [80-83], defined by a global scalar mass $m_{0}$ giving mass to all scalar particles, a gaugino mass $M_{1 / 2}$, a trilinear soft term $A_{0}$, tan $\beta$, the sign of $\mu$ and gauge coupling unification at the high scale $M_{\mathrm{GUT}}$. Apart from $\tan \beta$, all of these parameters are defined at the high scale $M_{\mathrm{GUT}}$. This model has 4 free parameters and the undefined sign of $\mu$. We will probe both signs of $\mu$.

- mSUGRA-var, defined mostly in the same way as mSUGRA except for one modification: we allow for free ratios of the gaugino masses such that $f_{1} M_{1}=f_{2} M_{2}=M_{3}=$ $M_{1 / 2}$. This model has 6 free parameters and the sign of $\mu$ that one can choose. The number of parameters that are assumed to be independent for the computation of the BG FT measure (see section 2.3) is the same as for the mSUGRA model. This model allows us to study the impact of HS model dependence.

- $N U H G M$ [84], where we use two independent mass parameters for the slepton and squark sector. We use $m_{0, L}$ as a soft-breaking SUSY mass parameter for all sparticles of the left-handed SM particles and $m_{0, R}$ for all sparticles of the right-handed SM particles. The gaugino masses are not required to unify and are given by three independent parameters: $M_{1}, M_{2}$ and $M_{3}$. Furthermore, there is one trilinear soft 
term $A_{0}$, the supersymmetric higgsino mass term $\mu, \tan \beta$ and finally the pseudoscalar Higgs boson pole mass $m_{A}$. We demand gauge coupling unification at the high scale $M_{\text {GUT }}$. All SUSY parameters, except $m_{A}, \mu$ and $\tan \beta$, are defined at the high scale $M_{\mathrm{GUT}}$. This model has 9 free parameters.

- $p M S S M-G U T$ [85], defined by the gaugino masses $M_{1}, M_{2}, M_{3}$, first/second generation scalar masses $m_{\tilde{Q}_{1}}, m_{\tilde{u}_{R}}, m_{\tilde{d}_{R}}, m_{\tilde{L}_{1}}, m_{\tilde{e}_{R}}$, third generation scalar masses $m_{\tilde{Q}_{3}}$, $m_{\tilde{t}_{R}}, m_{\tilde{b}_{R}}, m_{\tilde{L}_{3}}, m_{\tilde{\tau}_{R}}$, trilinear soft terms $A_{t}, A_{b}, A_{\tau}$, the pseudo-scalar Higgs boson pole mass $m_{A}$, the higgsino mass term $\mu$ and the ratio of weak scale Higgs vevs $\tan \beta$. All parameters, except $m_{A}, \mu$ and $\tan \beta$, are defined at the high scale $M_{\mathrm{GUT}}$, the scale where the coupling constants unify. This model has 19 free parameters. The model is very closely related to the pMSSM as defined above, and indeed the only difference is that in the pMSSM the parameters are defined at the low-energy SUSY breaking scale instead of the GUT scale. Since the number of LS parameters is the same as the number of HS ones, we can study the impact of the RGE running on the FT by defining the matching conditions at the HS.

In what follows, we will compute the minimal possible amount of FT that each of these models has after imposing all experimental constraints. To compute the amount of FT, we will use the EW FT measure and the BG FT measure, which are explained in more detail below.

\subsection{The electroweak fine-tuning measure}

The EW FT measure ( $\left.\Delta_{\mathrm{EW}}\right)$ was first proposed in ref. [10]. It parameterizes how sensitive $M_{Z}$ (eq. (2.1)) is to variations in each of the coefficients $C_{i}$ (as defined below). The measure is defined as

$$
\Delta_{\mathrm{EW}} \equiv \max _{i}\left|\frac{C_{i}}{M_{Z}^{2} / 2}\right|
$$

where the $C_{i}$ are

$$
\begin{aligned}
C_{m_{H_{d}}} & =\frac{m_{H_{d}}^{2}}{\tan ^{2} \beta-1}, & C_{m_{H_{u}}} & =\frac{-m_{H_{u}}^{2} \tan ^{2} \beta}{\tan ^{2} \beta-1}, \\
C_{\Sigma_{d}^{d}} & =\frac{\max \left(\Sigma_{d}^{d}\right)}{\tan ^{2} \beta-1}, & C_{\Sigma_{u}^{u}} & =\frac{-\max \left(\Sigma_{u}^{u}\right) \tan ^{2} \beta}{\tan ^{2} \beta-1} .
\end{aligned}
$$

The tadpole contributions $\Sigma_{u}^{u}$ and $\Sigma_{d}^{d}$ contain a sum of different contributions. All these contributions are computed individually and the maximum of these contributions is used to compute the $C_{\Sigma_{u}^{u}}$ and $C_{\Sigma_{d}^{d}}$ coefficients.

\subsection{The Barbieri-Giudice measure}

Another widely used measure is the BG measure proposed in refs. [14, 42]:

$$
\begin{aligned}
\Delta_{\mathrm{BG}} & \equiv \max \left|\Delta_{p}\right| \\
\Delta_{p} & \equiv \frac{\partial \ln M_{Z}^{2}}{\partial \ln p_{i}},
\end{aligned}
$$


where $p_{i}$ is one of the independent input parameters of the SUSY model. These input parameters can be defined at any scale. When the input parameters are defined at $M_{\text {SUSY }}$ we will use the notation $\Delta_{\mathrm{BG}}^{\mathrm{LS}}$. On the other hand, when the input parameters are defined at $M_{\mathrm{GUT}}$, which will be the case for all of the HS models that we will be considering in this paper, we will use the notation $\Delta_{\mathrm{BG}}^{\mathrm{HS}}$. Note that, in contrast to $\Delta_{\mathrm{EW}}$, the BG FT measure does take dependencies between the theory parameters into account. To use the BG FT measure, the LS parameters $m_{H_{u / d}}$ and $\mu$ that appear in eq. (2.1) need to be expressed as the fundamental parameters of the assumed SUSY model. These two sets of parameters are related by renormalization group equations (RGEs), which can be solved numerically. The dependence of the LS parameters on the input parameters takes the form of $[27,86]$

$$
\begin{aligned}
m_{H_{u}}^{2}\left(M_{\mathrm{SUSY}}\right)= & c_{M_{1}^{2}} M_{1}^{2}+c_{M_{2}^{2}} M_{2}^{2}+c_{M_{3}^{2}} M_{3}^{2}+c_{M_{1} M_{2}} M_{1} M_{2}+\ldots \\
& +c_{A_{t}^{2}} A_{t}^{2}+c_{A_{b}^{2}} A_{b}^{2}+\cdots+c_{A_{t} M_{3}} A_{t} M_{3}+\ldots \\
& +c_{m_{H_{u}}^{2}} m_{H_{u}}^{2}+c_{m_{\tilde{Q}_{3}}^{2}} m_{\tilde{Q}_{3}}^{2}+c_{m_{\tilde{t}_{R}}^{2}} m_{\tilde{t}_{R}}^{2}+c_{m_{\tilde{b}_{R}}^{2}} m_{\tilde{b}_{R}}^{2}+\ldots \\
m_{H_{d}}^{2}\left(M_{\mathrm{SUSY}}\right)= & c_{M_{1}^{2}} M_{1}^{2}+c_{M_{2}^{2}} M_{2}^{2}+c_{M_{3}^{2}} M_{3}^{2}+c_{M_{1} M_{2}} M_{1} M_{2}+\ldots, \\
& +c_{A_{t}^{2}} A_{t}^{2}+c_{A_{b}^{2}} A_{b}^{2}+\cdots+c_{A_{t} M_{3}} A_{t} M_{3}+\ldots \\
& +c_{m_{H_{d}}^{2}} m_{H_{d}}^{2}+c_{m_{\tilde{Q}_{3}}^{2}} m_{\tilde{Q}_{3}}^{2}+c_{m_{\tilde{t}_{R}}^{2}} m_{\tilde{t}_{R}}^{2}+c_{m_{\tilde{b}_{R}}^{2}} m_{\tilde{b}_{R}}^{2}+\ldots \\
\mu\left(M_{\mathrm{SUSY}}\right)= & c_{\mu} \mu,
\end{aligned}
$$

where the dots stand for contributions to the LS parameter that are similar to the ones already denoted. The parameters on the right-hand sides of these equations are input parameters defined at either $M_{\text {SUSY }}$ (in the case of the pMSSM) or at a HS. The numerical values of the coefficients $c_{i}$ depend on the values of the SM matching parameters (coupling constants and masses), the scale at which the SUSY input parameters are defined, $\tan \beta$ and the SUSY breaking scale.

\subsubsection{Dependence on high-scale model assumptions}

In this subsection, we will see an example of how the chosen set of fundamental parameters impact $\Delta_{\mathrm{BG}}^{\mathrm{HS}}$. To this end, consider $m_{H_{u}}^{2}\left(M_{\mathrm{SUSY}}\right)$ of eq. (2.5), where we now explicitly show the values of the numerical coefficients using $M_{\mathrm{SUSY}}=1 \mathrm{TeV}, \tan \beta=10$, a high-scale value of $10^{16} \mathrm{GeV}$ and the usual values for the SM input parameters $[27,86]$

$$
\begin{aligned}
m_{H_{u}}^{2}(1 \mathrm{TeV}) & \\
= & -1.603 M_{3}^{2}+0.203 M_{2}^{2}+0.006 M_{1}^{2}-0.005 M_{1} M_{2}-0.02 M_{1} M_{3}-0.134 M_{2} M_{3} \\
& -0.109 A_{t}^{2}+0.012 A_{t} M_{1}+0.068 A_{t} M_{2}+0.285 A_{t} M_{3}+0.001 A_{b}^{2}-0.002 A_{b} M_{3} \\
& +0.631 m_{H_{u}}^{2}-0.367 m_{\tilde{Q}_{3}}^{2}-0.025 m_{\tilde{Q}_{2}}^{2}-0.025 m_{\tilde{Q}_{1}}^{2}-0.290 m_{\tilde{t}_{R}}^{2} \\
& +0.054 m_{\tilde{c}_{R}}^{2}+0.054 m_{\tilde{u}_{R}}^{2}-0.024 m_{\tilde{b}_{R}}^{2}-0.025 m_{\tilde{s}_{R}}^{2}-0.025 m_{\tilde{d}_{R}}^{2}+0.026 m_{H_{d}}^{2} \\
& -0.026 m_{\tilde{\tau}_{R}}^{2}-0.026 m_{\tilde{\mu}_{R}}^{2}-0.026 m_{\tilde{e}_{R}}^{2}+0.025 m_{\tilde{L}_{3}}^{2}+0.025 m_{\tilde{L}_{2}}^{2}+0.025 m_{\tilde{L}_{1}}^{2} \\
& +\ldots,
\end{aligned}
$$

where the dots indicate less important contributions. Here, one can observe that the gluino mass parameter $M_{3}$ and the soft SUSY breaking mass parameters $m_{\tilde{Q}_{3}}, m_{\tilde{t}_{R}}$ and 
$m_{H_{u}}$ will greatly contribute to the value of $\Delta_{\mathrm{BG}}^{\mathrm{HS}}$, as their coefficients are relatively large. To demonstrate the dependence of $\Delta_{\mathrm{BG}}^{\mathrm{HS}}$ on the input parameters, consider the contribution of $m_{\tilde{Q}_{3}}$ to the FT

$$
\begin{aligned}
\Delta_{m_{\tilde{Q}_{3}}} & =\frac{m_{\tilde{Q}_{3}}}{M_{Z}^{2}} \frac{\partial M_{Z}^{2}}{\partial m_{\tilde{Q}_{3}}} \\
& \simeq-2 \frac{m_{\tilde{Q}_{3}}}{M_{Z}^{2}} \frac{\partial m_{H_{u}}^{2}}{\partial m_{\tilde{Q}_{3}}}=-4 c_{m_{\tilde{Q}_{3}}^{2}} \frac{m_{\tilde{Q}_{3}}^{2}}{M_{Z}^{2}}=1.468 \frac{m_{\tilde{Q}_{3}}^{2}}{M_{Z}^{2}} .
\end{aligned}
$$

Here, one sees that a large value for $m_{\tilde{Q}_{3}}$ at the GUT scale will automatically lead to a large value for $\Delta_{m_{\tilde{Q}_{3}}}$. The same holds true for $M_{3}, m_{\tilde{t}_{R}}$ and $m_{H_{u}}$, hence the wide-spread assumption that gluinos and stops should be light to avoid large fine-tunings. Note that here one can already observe the strong dependence of $\Delta_{\mathrm{BG}}^{\mathrm{HS}}$ on the model assumptions, as there is a factor of 2 difference in taking $m_{\tilde{Q}_{3}}$ or $m_{\tilde{Q}_{3}}^{2}$ as a fundamental (input) parameter

$$
\Delta_{m_{\tilde{Q}_{3}}^{2}}=\frac{m_{\tilde{Q}_{3}}^{2}}{M_{Z}^{2}} \frac{\partial M_{Z}^{2}}{\partial m_{\tilde{Q}_{3}}^{2}} \simeq 0.734 \frac{m_{\tilde{Q}_{3}}^{2}}{M_{Z}^{2}} .
$$

This shows that the overall amount of FT is reduced by a factor of 2 if the input parameters squared are taken as fundamental parameters. In the foregoing, we have assumed that all pMSSM parameters are independent at the high scale. To explicitly see how parameter dependencies impact the value for $\Delta_{\mathrm{BG}}^{\mathrm{HS}}$, we can impose a relation between several input parameters

$$
\begin{aligned}
& m_{\tilde{Q}_{3}}=m_{H_{u}}=m_{H_{d}}=m_{\tilde{t}_{R}}=\ldots \equiv m_{0}, \\
& M_{1}=M_{2}=M_{3} \equiv M_{1 / 2}, \\
& A_{t}=A_{b} \equiv A_{0} .
\end{aligned}
$$

This reduces the numerical values of the RGE coefficients. Using the code of ref. [27], we now obtain

$$
m_{H_{u}}^{2}(1 \mathrm{TeV})=-1.553 M_{1 / 2}^{2}-0.108 A_{0}^{2}+0.362 M_{1 / 2} A_{0}-0.018 m_{0}^{2} .
$$

The contribution stemming from the scalar soft SUSY breaking masses to $\Delta_{\mathrm{BG}}^{\mathrm{HS}}$ is now greatly reduced compared to the previous example where all parameters were independent, as the highest value for all scalar coefficients (previously $c_{m_{H_{u}}}$ ) drops with a factor of $\sim 7$ in the new coefficient $c_{m_{0}}$. Of course, this unification is well known and exactly defines the unification assumed in the mSUGRA model as described in the previous section [80-83].

The goal of the foregoing was to show that $\Delta_{\mathrm{BG}}^{\mathrm{HS}}$ will depend greatly on the chosen parameter dependencies and the chosen set of fundamental parameters. This means that two different high-scale SUSY models with exactly the same mass spectra can lead to radically different values of $\Delta_{\mathrm{BG}}^{\mathrm{HS}}$, depending on the HS model assumptions, like noted before in e.g. refs. [75, 76, 87]. Furthermore, in ref. [88] it was shown that conclusions drawn from $\Delta_{\mathrm{BG}}^{\mathrm{HS}}$ (or $\Delta_{\mathrm{BG}}^{\mathrm{LS}}$ ) are very sensitive to the order of accuracy of the RGE equations. 
The EW FT measure $\Delta_{\mathrm{EW}}$ as given in eq. (2.2) is not affected by these assumptions and should therefore be seen as a more conservative measure, although it is strictly only applicable to an LS SUSY model such as the pMSSM. It is evaluated from weak scale parameters containing no information on a possible HS theory. Therefore it gives only an indicative FT value for a given EW spectrum interpreted in the pMSSM. We can interpret a small value of $\Delta_{\mathrm{EW}}$ for a given SUSY spectrum as a minimal necessary condition of a natural SUSY model, but it is not a sufficient one, as we will also see in what follows. The real value of FT will depend on the exact parameter conditions that are present for the HS SUSY model and we will see in section 4.4 that this can be either higher or lower for some spectra. Note that the two measures can also agree for specific HS model assumptions and values for the input parameters. This may happen for example when both the EW and BG FT measures are dominated by the value of $\mu$. As the value for $c_{\mu}$ is close to one, in this case $\Delta_{\mathrm{EW}} \simeq \Delta_{\mathrm{BG}}^{\mathrm{HS}}$ if $\mu^{2}$ is chosen as a fundamental parameter.

\subsection{Concluding remarks}

In this section we have shown the differences between two popular FT measures used to quantify FT in SUSY models: $\Delta_{\mathrm{EW}}$ (eq. (2.2)) and $\Delta_{\mathrm{BG}}^{\mathrm{HS}}$ (eq. (2.3)). The FT measure $\Delta_{\mathrm{BG}}^{\mathrm{HS}}$ suffers from model dependence more than $\Delta_{\mathrm{EW}}$, as the former is extremely sensitive to which HS model and set of fundamental parameters is chosen. This leads to the confusing notion that different models with exactly the same LS spectra can give rise to different values for $\Delta_{\mathrm{BG}}^{\mathrm{HS}}$. As we do not know the exact SUSY breaking mechanism or HS model assumptions, we should be careful in using $\Delta_{\mathrm{BG}}^{\mathrm{HS}}$ to construct natural mass ranges for sparticles or conclude anything about the exclusion of a natural realization of the MSSM. On the other hand, $\Delta_{\mathrm{EW}}$ suffers from the fact that it can only indicate a conservative value for the FT in a given LS spectrum. To compute the actual amount of FT, one needs to construct a HS theory that can give rise to the same LS spectrum and recompute the FT taking into account all parameter dependencies. Often it is quoted that $\Delta_{\mathrm{EW}}$ will give a lower bound on the fine-tuning of any HS model with a given SUSY spectrum (see e.g. refs. $[75,87])$. However, we will see that in certain situations, this statement is not true. To clearly highlight the different conclusions one can draw using both FT measures, in what follows we will minimize these measures in the four different SUSY GUT scenarios that have been described in section 2.1, taking into account all current constraints and future experiments, which will be described in the following section.

\section{Analysis setup}

Already a minimal model such as the pMSSM has a very rich phenomenology. Therefore, it is necessary to intelligently scan the parameter space, probing it for interesting regions, which could be missed if one adopts a random scan using, for instance, flat priors, which is often done in the fine-tuning literature. We use the Gaussian particle filter [89] to tackle this problem. This scanning algorithm starts off by collecting an initial seed of randomly generated points. Then, in an iterative procedure, the best-fit points of the foregoing iteration are used as seeds to sample new model points, where a multi-dimensional Gaussian 
distribution is used around each seed parameter. The width of the Gaussian distribution in a specific dimension is chosen to be a variable fraction times the value of the seed point in that dimension. The fraction depends on the stage of the iteration. In the beginning, the fraction is chosen to be large (1-2) in order to be sensitive to a wide range of values. However, if one finds an interesting and possibly narrow region in the parameter space, the fraction needs to be reduced in order to efficiently probe it. In total, we have generated around $\mathcal{O}(100)$ million spectra for each GUT scale model.

To create the SUSY spectra we use SofTSUSY 4.0 [90], while the Higgs mass is calculated using FeynHiggs 2.14 .2 [91-95]. We only select models that have the lightest neutralino $\tilde{\chi}_{1}^{0}$ as lightest supersymmetric particle (LSP) and discard spectra with tachyons or that do not satisfy the EWSB conditions. SUSYHIT [96] is used to calculate the decay of the SUSY and Higgs particles.

MicrOMEGAs 4.3.4 [97] is used to compute several flavor variables, the muon anomalous magnetic moment, the dark matter relic density $\left(\Omega_{\mathrm{DM}} h^{2}\right),{ }^{2}$ the present-day velocityweighted annihilation cross section $(\langle\sigma v\rangle)$ and the spin-dependent and spin-independent WIMP-nucleon scattering cross sections $\left(\sigma_{\mathrm{SD}}\right.$ and $\left.\sigma_{\mathrm{SI}}\right)$. The constraints on the WIMPnucleon scattering cross sections stemming from various dark matter direct detection (DMDD) experiments are computed using DDCALC 2.0.0 [99] where the 2018 and 2019 results from XENON1T [100, 101], the 2017 and 2019 limits from PICO [102-104] and the 2018 limits from PandaX [105, 106] are implemented. For DM indirect detection we only consider the limit on $\langle\sigma v\rangle$ stemming from the observation of gamma rays originating from dwarf galaxies, which we implement as a hard cut on each of the channels reported on the last page of ref. [107]. Other DM indirect detection experiments are not taken into account as they are found not to constrain the DM properties any further. We use the central values of MicrOMEGAs 4.3.4 for the nuclear form factors, the DM local density and velocity. We allow for a multi-component DM, therefore the DM direct detection limits are rescaled by $f=\frac{\Omega h_{\mathrm{DM}}^{2}}{\Omega h_{\text {Planck }}^{2}}$ (or $f^{2}$ in the case of indirect detection) if the dark matter relic abundance is less than the observed value $\Omega h_{\text {Planck }}^{2}=0.120 \pm 0.001$ [108].

We use SUSY-AI to determine the exclusion of a model point in the pMSSM parameter space based on the ATLAS $13 \mathrm{TeV}$ results $[109,110]$. To cross check the SUSY-AI results, we have used SMODELS [111-115] for a selection of the models that have the lowest FT. HigGsBound 5.1.1 is used to determine whether the SUSY models satisfy the LEP, Tevatron and LHC Higgs constraints [116-123]. Vevacious [124-126] is used to check that the models do not have a color/charge breaking minimum and have at least a meta-stable minimum that has a lifetime that exceeds that of our Universe.

We apply the following cuts on the values for certain masses and flavor observables:

- LEP limits on the masses of the chargino $\left(m_{\tilde{\chi}_{1}^{ \pm}}>103.5 \mathrm{GeV}\right)$ and light sleptons $\left(m_{\tilde{l}}>90 \mathrm{GeV}\right)$ [127]. For the staus we use a limit of $m_{\tilde{\tau}}>85 \mathrm{GeV}$. We do not save any spectra that have sparticle masses below these limits.

- Constraints on the invisible and total width of the $Z$-boson $\left(\Gamma_{Z, \text { inv }}=499.0 \pm 1.5 \mathrm{MeV}\right.$ and $\left.\Gamma_{Z}=2.4952 \pm 0.0023 \mathrm{GeV}\right)[128]$.

\footnotetext{
${ }^{2}$ The computed values for $\Omega h_{\mathrm{DM}}^{2}$ were cross-checked with MicROMEGAs 5.0.9 [98].
} 


\begin{tabular}{|c|c|c|c|}
\hline Particle & Mass cut (HL-LHC) & Mass cut (HE-LHC) & Mass cut (CLIC) \\
\hline$\tilde{g}$ & $3.2 \mathrm{TeV}$ & $5.7 \mathrm{TeV}$ & - \\
$\tilde{t}_{1}$ & $1.7 \mathrm{TeV}$ & $3.6 \mathrm{TeV}$ & - \\
$\tilde{\chi}_{1}^{ \pm}$(higgsino) & $350 \mathrm{GeV}$ & $550 \mathrm{GeV}$ & $1.5 \mathrm{TeV}$ \\
$\tilde{\tau}_{1}$ & $730 \mathrm{GeV}$ & $1.15 \mathrm{TeV}$ & - \\
\hline
\end{tabular}

Table 1. Exclusion potential of the HL-LHC, HE-LHC and CLIC on various SUSY particles as implemented in this analysis. The values for the HL-LHC and HE-LHC are taken from ref. [137]. For CLIC [138] we simply divided the optimal energy reach of $3 \mathrm{TeV}$ by 2 as a baseline.

- The lightest Higgs boson is required to be in the mass range of $122 \mathrm{GeV} \leq m_{h_{0}} \leq$ $128 \mathrm{GeV}$.

- Taking into account the fact that the SM prediction lies well outside the experimentally obtained value with a discrepancy of $\Delta(g-2)_{\mu}=(24.9 \pm 6.3) \times 10^{-10}$ [129], we allow for spectra that predict $\Delta(g-2)_{\mu}<40 \times 10^{-10}$.

- Measurements of the $B / D$-meson branching fractions $\operatorname{Br}\left(B_{s}^{0} \rightarrow \mu^{+} \mu^{-}\right)$[130], $\operatorname{Br}\left(\bar{B} \rightarrow X_{s} \gamma\right)$ [131, 132], $\operatorname{Br}\left(B^{+} \rightarrow \tau^{+} \nu_{\tau}\right)$ [133], $\operatorname{Br}\left(D_{s}^{+} \rightarrow \mu^{+} \nu_{\mu}\right)$ [134] and $\operatorname{Br}\left(D_{s}^{+} \rightarrow \tau^{+} \nu_{\tau}\right)[135]$.

For all observables (except for the LEP limits, $m_{h_{0}}, \Delta(g-2)_{\mu}$ and the SUSY-AI outputs) we require the value to lie within a $3 \sigma$ interval from the observed value. Firstly, we do this to be conservative, and not disregard true models too easily. Secondly, some of these limits, especially the DMDD constraints, are subject to large uncertainties. As it is beyond the scope of this project to fully model these uncertainties, we allow for a looser criterion on when we disregard a spectrum, as was done before in e.g. [32, 136].

The EW FT measure $\Delta_{\mathrm{EW}}$ is calculated by computing the effective potential terms and determining the maximal contribution via eq. (2.2), using the code from ref. [32]. Note that this code differs from the built-in function from SofTSUSY 4.0 in how it handles the tadpole terms. While the latter code sums up all contributions in the tadpole terms and then computes the FT, we take the maximum value of each term in the tadpole. This ensures that we don't have a large cancellation in e.g. the stop sector, which could result in a very low value for $\Delta_{\mathrm{EW}}$. An explicit example of this mechanism can be seen in section 4.4. The BG measure of eq. (2.3) is calculated via the procedure implemented in SoftSUSY 4.0. ${ }^{3}$

\subsection{Implementation of the limits imposed by future experiments}

In this study we will consider two kinds of future experiments: DMDD experiments and colliders, as these two classes of experiments will have the biggest impact on the pMSSM parameter space.

\footnotetext{
${ }^{3} \mathrm{An}$ error was found in the calculation of the tadpole contributions to the fine-tuning, so we corrected the error in our version of SoftSUSY. This was communicated with the authors and updated in newer versions of SoftSUSY.
} 
Future DMDD experiments that are considered are the LZ experiment [139], DARWIN [140, 141], Darkside-50k [142] and the PICO-500 experiment [143]. The first three experiments are most useful for constraining the SI WIMP-neutron scattering cross sections, while PICO-500 is more sensitive to the SD WIMP-proton scattering cross section. These detectors and analyses are implemented in the code DDCALC [99], which is used to calculate the exclusion limits. The limits depend on $\sigma_{\mathrm{SD}}$ and $\sigma_{\mathrm{SI}}$, the dark matter local density and velocity and the nuclear form factors assumed. We do not consider future DM indirect detection experiments as their exclusion power is not as powerful as that of the DMDD experiments.

The predicted exclusion reach for collider experiments is implemented simply as a mass cut on the relevant mass parameter. To set the exclusions reaches we follow ref. [137], where the sensitivity of the High-Luminosity (HL) and High-Energy (HE) phase of the LHC on SUSY particles is discussed. The mass limits that we will use are given in table 1. Given the sensitivity of the higgsino mass parameter on the amount of FT, we also add the reach of the Compact Linear Collider (CLIC) [138] as its maximal energy reach (3 TeV) divided by 2 . Note that this is a simplified approach: in reality, the limits on the masses may be lower depending on the exact SUSY spectrum and the complexity of the decays of the SUSY particles. We stress that these simple mass limits are only an indication of how far the future HL and HE-LHC can maximally reach. For the electroweak sparticles, we only implement the higgsino mass limits in this analysis, as the value of the wino mass parameter has little impact on the amount of FT.

\section{Results}

In this section we report on the resulting minimum allowed amount of FT, using either the BG FT measure $\Delta_{\mathrm{BG}}^{\mathrm{HS}}$ (defined in eq. (2.3)) or the EW FT measure $\Delta_{\mathrm{EW}}$ (defined in eq. (2.1)). As explained in section 2, we will report our results for four different HS SUSY models, whose spectra all are embedded in the pMSSM. We will start with the mSUGRA model, then move on to mSUGRA-var, then consider NUHGM and finally look at the pMSSM-GUT model. We will also consider some phenomenology of the spectra that have the lowest FT for both measures, and their prospects to be probed at future experiments. Every sub-section will be structured in the following way: first, we will discuss the current status of $\Delta_{\mathrm{BG}}^{\mathrm{HS}}$. Then we move on to $\Delta_{\mathrm{EW}}$, combined with a discussion on the impact of current and future DMDD experiments. Every sub-section will end with a discussion on the future collider prospects.

\section{1 mSUGRA}

The resulting values for $\Delta_{\mathrm{BG}}^{\mathrm{HS}}$ and $\Delta_{\mathrm{EW}}$ for all generated mSUGRA spectra are shown in figure 1 as a function of the dark matter relic density $\Omega h^{2}$. The lowest value for $\Delta_{\mathrm{BG}}^{\mathrm{HS}}$ is 571. The minimal value for $\Delta_{\mathrm{BG}}^{\mathrm{HS}}$ is constrained mainly by the Higgs mass requirement, and to a lesser extent by the limit placed on $\operatorname{Br}\left(B_{s} \rightarrow \mu^{+} \mu^{-}\right)$. Dropping the Higgs mass requirement, while keeping all other constraints, would result in a value for $\Delta_{\mathrm{BG}}^{\mathrm{HS}}$ of about 240. The region where $\Delta_{\mathrm{BG}}^{\mathrm{HS}}$ is minimized corresponds to values of $M_{1 / 2} \simeq 800 \mathrm{GeV}$, 
$A_{0} \simeq-3 \mathrm{TeV}$ and $m_{0} \simeq 2.5 \mathrm{TeV}$. The value for $\tan \beta$ is less constrained and lies between 10 and 50 in this region. There is a clear reason why these specific values for $m_{0}, A_{0}$ and $M_{1 / 2}$ are preferred. If the value for $m_{0}$ is lowered, $|\mu|$ needs to increase to still satisfy the EWSB requirement, so the amount of fine-tuning is increased because of $|\mu|$. If $m_{0}$ is increased, $\Delta_{\mathrm{BG}}^{\mathrm{HS}}$ increases due to $m_{0}$ [75]. The Higgs mass requirement prevents $M_{1 / 2}$ and $A_{0}$ to get lower, although lowering the absolute value of these two parameters would result in a lower $\Delta_{\mathrm{BG}}^{\mathrm{HS}}$. Another reason why low values of $M_{1 / 2}$ are not allowed is the limit placed on $\operatorname{Br}\left(B_{s} \rightarrow \mu^{+} \mu^{-}\right)$, as it leads to a too light pseudo-scalar Higgs boson. Hence we find a special region where $\Delta_{\mathrm{BG}}^{\mathrm{HS}}$ is minimized, driven mainly by the observed value of the Higgs mass.

This region can also be observed in figure 2, where on the left-hand side the gluino mass $\left(m_{\tilde{g}}\right)$ is plotted against the lightest stop mass $\left(m_{\tilde{t}_{1}}\right)$. The value for $\Delta_{\mathrm{BG}}^{\mathrm{HS}}$ is shown as a color code, whose minimum is reached for gluino and stop masses of $\mathcal{O}(2 \mathrm{TeV})$. One observes that $\Delta_{\mathrm{BG}}^{\mathrm{HS}}$ increases for higher stop and gluino masses. Both $m_{H_{u}}$ and $m_{H_{d}}$ depend on the gaugino mass parameter $M_{1 / 2}$ with a large RGE coefficient. Therefore, $M_{1 / 2}$ needs to be as low as experimentally allowed in order to keep the value for $\Delta_{\mathrm{BG}}^{\mathrm{HS}}$ as small as possible. A higher value for $M_{1 / 2}$ will result in a higher value for both $m_{\tilde{g}}$ and $m_{\tilde{t}_{1}}$. Therefore, by increasing $m_{\tilde{g}}$ and $m_{\tilde{t}_{1}}$, one also sees that the value for $\Delta_{\mathrm{BG}}^{\mathrm{HS}}$ is increased.

In the neutralino-chargino sector, there is not much freedom for the allowed masses due to the unification of the gaugino masses, as can be seen on the right-hand side of figure 2 . The gaugino mass parameter $M_{1 / 2}$ needs to be large at the GUT scale for the allowed spectra to satisfy the observed value for the Higgs boson mass, evade the gluino mass limits and the limit on $\operatorname{Br}\left(B_{s} \rightarrow \mu^{+} \mu^{-}\right)$. It is well known that the ratio of the gaugino masses at the SUSY scale is roughly $M_{3} \simeq 2.7 M_{2} \simeq 5 M_{1}$ due to the unification of the gaugino masses at the GUT scale, where the exact ratio depends on the numerical value of the GUT and SUSY scale. Due to this relation, $M_{1}$ is prevented to get lower than about $200 \mathrm{GeV}$ at the SUSY scale, otherwise, the gluino mass would also get too. LSPs with a mass around $100 \mathrm{GeV}$ can then only be higgsino-like and are necessarily accompanied by a higgsino-like chargino with a similar mass. For slightly higher LSP masses, the LSP is also allowed to be bino-like. For these models, the chargino will be wino-like with a higher mass than the LSP. This explains the presence of the two hard lines in figure 4. Spectra with mixed LSP compositions lie in-between these two hard lines. The value for $\Delta_{\mathrm{BG}}^{\mathrm{HS}}$ is minimized in the second region where the LSP is bino-like.

$\Delta_{\text {EW }}$ and the impact of DMDD experiments. While the observed value of the Higgs boson mass constrains the minimal value for $\Delta_{\mathrm{BG}}^{\mathrm{HS}}$, limits placed by current DMDD experiments constrain the minimal value for $\Delta_{\mathrm{EW}}$, which is 38 . This can be seen on the right-hand side of figure 1 . The minimal value that we find for $\Delta_{\mathrm{EW}}$ in the mSUGRA model is a factor of 10 lower than the ones reported previously in e.g. refs. [20, 76]. The spectra that minimize $\Delta_{\text {EW }}$ all feature a higgsino-dominated LSP with a negligible wino component, a small bino component $(<10 \%)$ and a mass of around $100-400 \mathrm{GeV}$. These spectra result in values for $\Omega h^{2}$ around $10^{-3}-10^{-2}$, where $\Omega h^{2}$ increases with higher values for $m_{\tilde{\chi}_{1}^{0}}$ and/or a larger bino component of $m_{\tilde{\chi}_{1}^{0}}$. The size of the SD cross section is proportional 

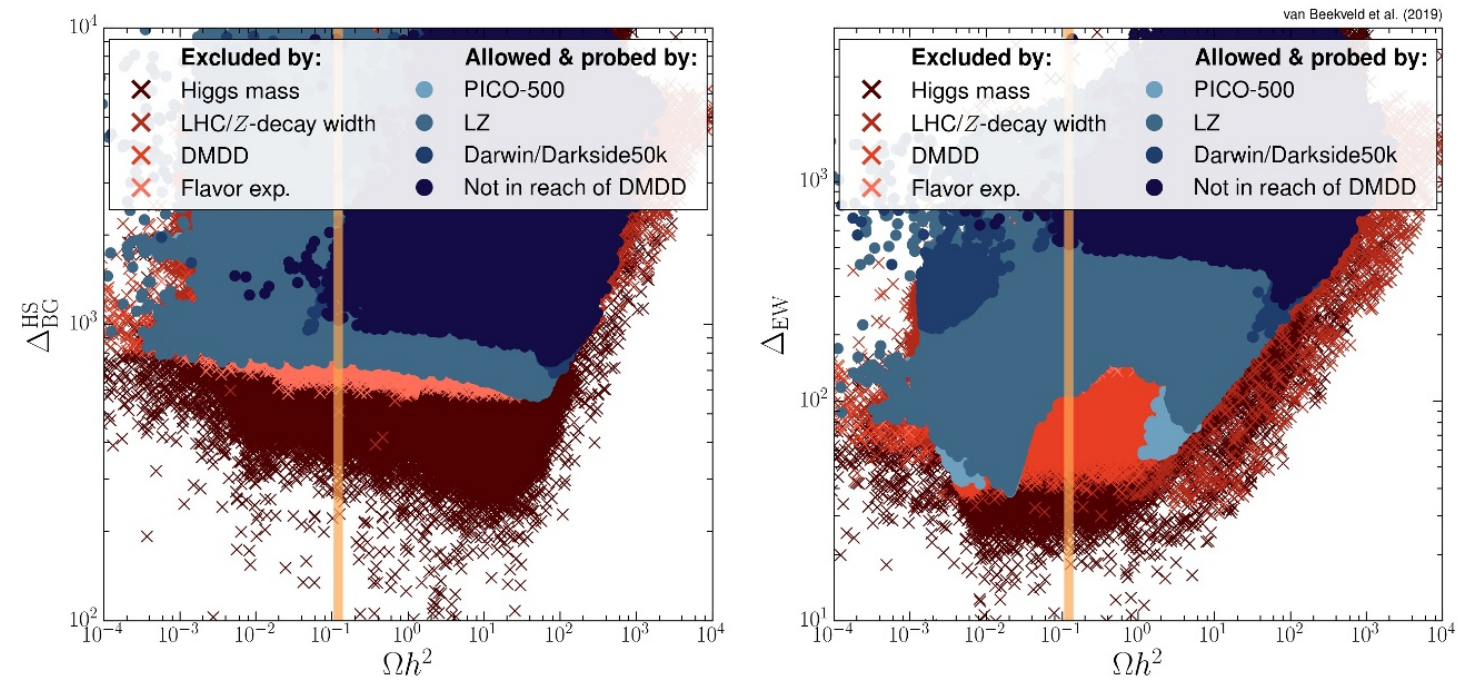

Figure 1. Generated spectra for the mSUGRA model. The left figure shows $\Delta_{\mathrm{BG}}^{\mathrm{HS}}$ as a function of $\Omega h^{2}$, the right figure shows $\Delta_{\mathrm{EW}}$ as a function of $\Omega h^{2}$. Red crosses indicate that the spectrum is excluded due to (in plotting order): the Higgs mass requirement (darkest red); limits placed on SUSY masses; by DMDD experiments; by limits placed on several flavor physics observables (lightest red) respectively. The constraints are also checked in this order. Circles in different shades of blue indicate the sensitivity of DMDD experiments, where in order of decreasing brightness we indicate: PICO-500, LZ, Darwin/Darkside50k or unconstrained by DMDD experiments. Unconstrained spectra lie on top of more constrained and excluded spectra. The exclusions checks are only performed once in the indicated order, The orange band indicates $\Omega h^{2}=0.12$.
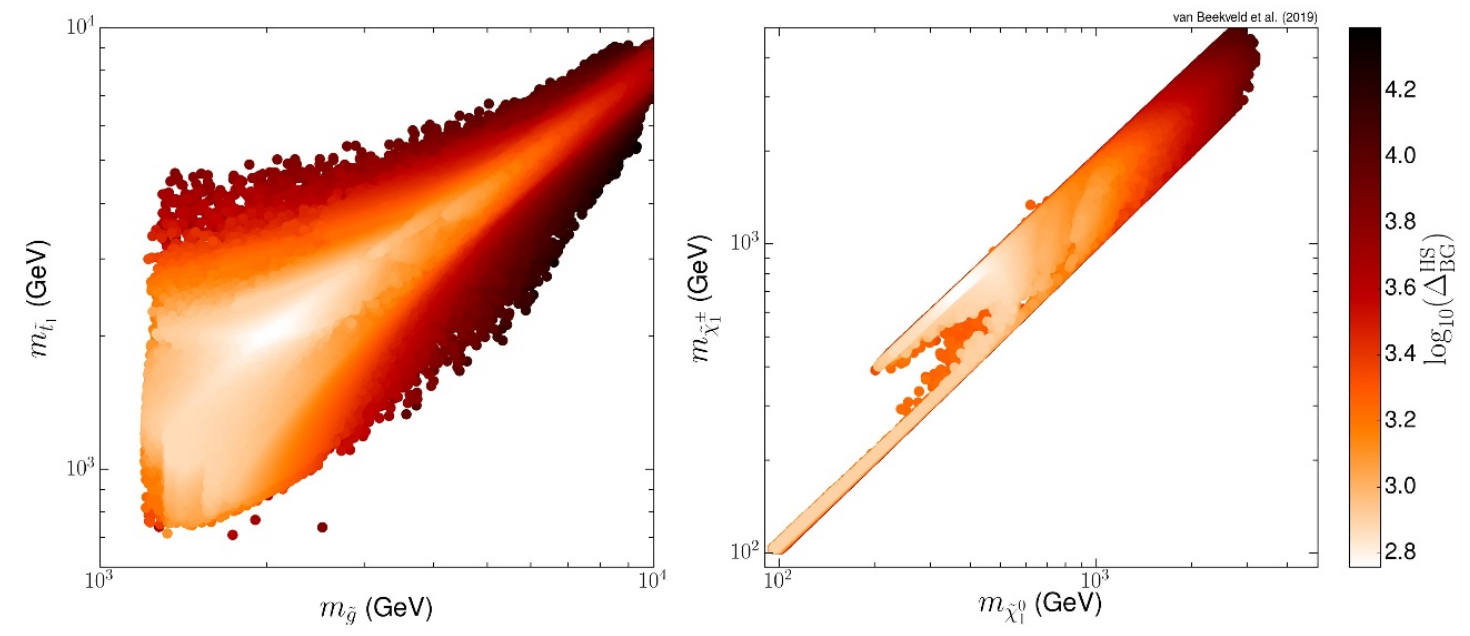

Figure 2. Left: the gluino mass $\left(m_{\tilde{g}}\right)$ against the lightest stop mass $\left(m_{\tilde{t}_{1}}\right)$ for mSUGRA, showing only the allowed spectra. Right: the lightest chargino mass $\left(m_{\tilde{\chi}_{1}^{ \pm}}\right)$against the lightest neutralino mass $\left(m_{\tilde{\chi}_{1}^{0}}\right)$ for the allowed spectra. All the masses are shown in units of GeV. The color code indicates the value for $\log _{10}\left(\Delta_{\mathrm{BG}}^{\mathrm{HS}}\right)$. Spectra with lower values for $\log _{10}\left(\Delta_{\mathrm{BG}}^{\mathrm{HS}}\right)$ lie on top of spectra with higher values. 


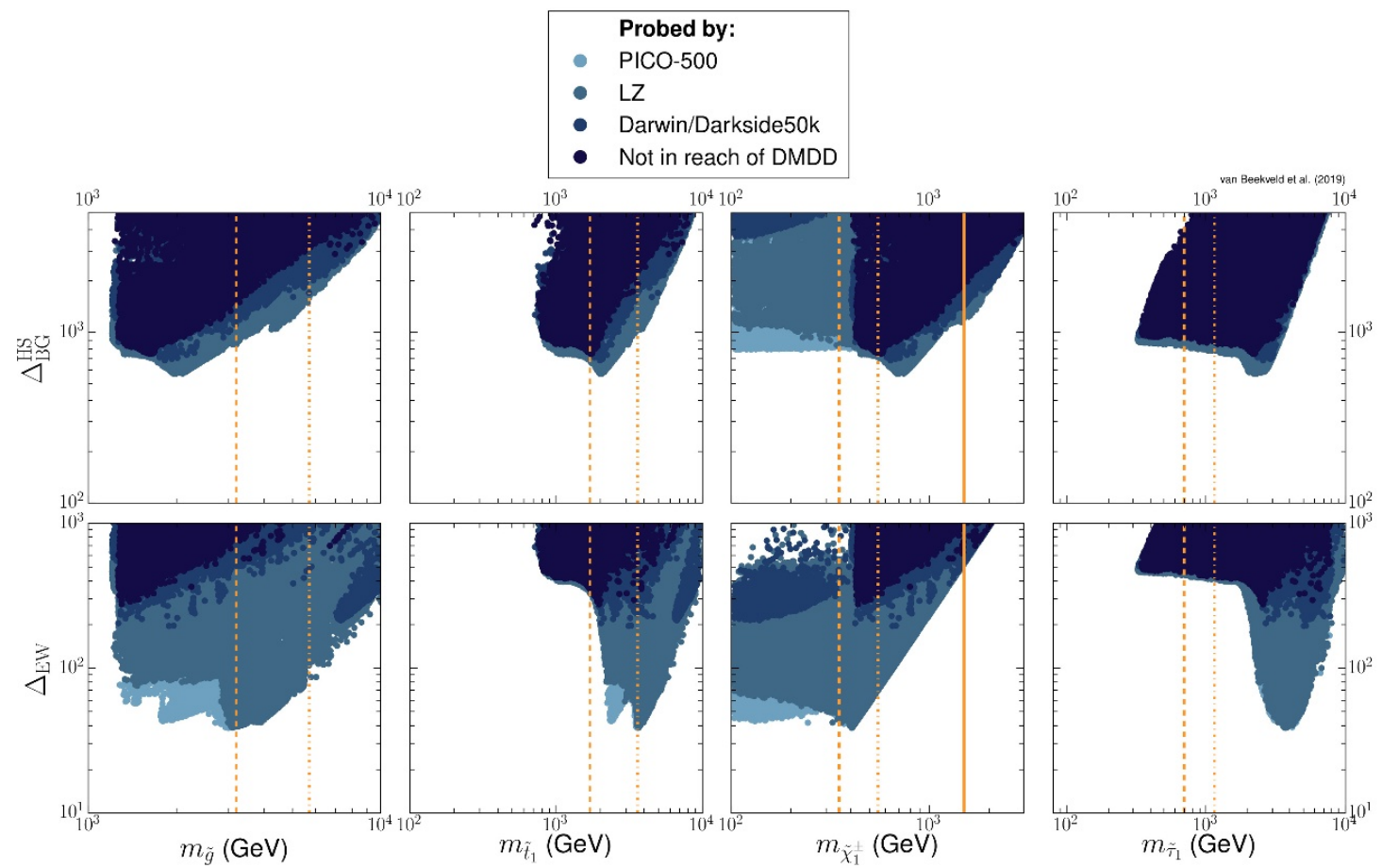

Figure 3. The values for $\Delta_{\mathrm{BG}}^{\mathrm{HS}}$ (top) and $\Delta_{\mathrm{EW}}$ (bottom) against (from left to right) the gluino mass $\left(m_{\tilde{g}}\right)$, stop mass $\left(m_{\tilde{t}_{1}}\right)$, chargino mass $\left(m_{\tilde{\chi}_{1}^{ \pm}}\right)$and stau mass $\left(m_{\tilde{\tau}_{1}}\right)$ for the allowed mSUGRA spectra. The masses are given in units of $\mathrm{GeV}$. The color code and plotting order is the same as in figure 1. The dashed, dash-dotted and solid orange line shows the exclusion potential of the HL-, HE-LHC and CLIC on the masses of various SUSY particles (see table 1). The solid orange line in the chargino mass plot shows the exclusion potential of CLIC.

to the $\tilde{\chi}_{1}^{0} \tilde{\chi}_{1}^{0} Z$ coupling, which is proportional to the difference between the two higgsino components of the LSP $\left(\left|N_{13}\right|^{2}-\left|N_{14}\right|^{2}\right)$. There is a higgsino asymmetry $\left(\left|N_{13}\right| \neq\left|N_{14}\right|\right)$ for these higgsino-dominated LSPs, therefore the $\tilde{\chi}_{1}^{0} \tilde{\chi}_{1}^{0} Z$ coupling is generally high. For this reason, these spectra will be fully probed by future DMDD experiments, despite the suppression factor that they receive due to the fact that $\Omega h^{2}<0.12$.

Around $\Omega h^{2} \gtrsim 0.1$, the LSPs become bino dominated with a small higgsino component. These models correspond to the models in-between the lower and upper band in figure 2, where it can be seen that the LSPs have masses above $300 \mathrm{GeV}$. In this regime, the DMDD experiments that are sensitive to either the SI or SD cross-sections constrain $\Delta_{\mathrm{EW}}$. At even higher values of $\Omega h^{2}$ the LSP will be a pure bino, so the DMDD experiments lose their sensitivity in this regime. The higgsino component keeps decreasing for higher $\Omega h^{2}$, which is the cause for the increase of $\Delta_{\mathrm{EW}}$ for $\Omega h^{2}>5$. The impact of future DMDD experiments is sizable, as these increase the minimal value for $\Delta_{\mathrm{EW}}$ to 275 . For models that have $0.09<\Omega h^{2}<0.15$, the minimal value for $\Delta_{\text {EW }}$ is around 515 . On the other hand, the minimal value of $\Delta_{\mathrm{BG}}^{\mathrm{HS}}$ can be increased to 750 by the reach of future DMDD experiments. 
The impact of future collider experiments. The dependence of $\Delta_{\mathrm{BG}}^{\mathrm{HS}}$ and $\Delta_{\mathrm{EW}}$ on $m_{\tilde{g}}, m_{\tilde{t}_{1}}$, lightest chargino mass $\left(m_{\tilde{\chi}_{1}^{ \pm}}\right)$and lightest stau mass $\left(m_{\tilde{\tau}_{1}}\right)$ is shown in figure 3 . We also show the reach of the future HL-LHC, HE-LHC and CLIC experiments as a dashed, dash-dotted and solid orange line respectively. One observes that the HL-HLC can bring the minimal value for $\Delta_{\mathrm{BG}}^{\mathrm{HS}}$ from 571 to about 848 by its power to constrain $m_{\tilde{g}}$. Due to its increased energy reach, the HE-LHC can bring the minimal value for $\Delta_{\mathrm{BG}}^{\mathrm{HS}}$ to about 1700 . The HE-LHC machine will be the most constraining for $\Delta_{\mathrm{BG}}^{\mathrm{HS}}$, as CLIC can constrain $\Delta_{\mathrm{BG}}^{\mathrm{HS}}$ to about 1240 in the case of a non-observation. The impact on $\Delta_{\mathrm{EW}}$ is less sizeable and has two origins, namely the exclusion reach of the HL and HE-LHC on both $m_{\tilde{g}}$ and $m_{\tilde{\chi}_{1}^{ \pm}}$. The HL-LHC increases the minimal value for $\Delta_{\mathrm{EW}}$ to about 38, while the HE-LHC increases the value to about 86 . The impact of CLIC on $\Delta_{\mathrm{EW}}$ is significant, as it can increase the current limit of $\Delta_{\mathrm{EW}}$ to about 530 .

\section{2 mSUGRA-var}

This model is closely related to the mSUGRA model that was considered in the previous section, but differs in the fact that it has more freedom in the gaugino sector. This feature is directly reflected in the minimal values for both of the FT measures. We will again first discuss $\Delta_{\mathrm{BG}}^{\mathrm{HS}}$, whose value as a function of $\Omega h^{2}$ can be seen on the left-hand side of figure 4. The lowest allowed value for $\Delta_{\mathrm{BG}}^{\mathrm{HS}}$ is $191,{ }^{4}$ which is a decrease of around 400 when compared to the mSUGRA model. The cause of this big decrease in $\Delta_{\mathrm{BG}}^{\mathrm{HS}}$ is that we treat $M_{1 / 2}$ as the only independent parameter in the FT computation, and at the same time let the ratios of $M_{1}$ and $M_{2}$ to $M_{3}$ be unconstrained. This shows an explicit example of the aforementioned dependence of $\Delta_{\mathrm{BG}}^{\mathrm{HS}}$ on the assumed dependencies that are present in the model. The Higgs mass requirement is again the strongest constraint for the minimum value of $\Delta_{\mathrm{BG}}^{\mathrm{HS}}$. Dropping this requirement, while keeping all of the other current collider and DM constraints, shows a decrease of $\Delta_{\mathrm{BG}}^{\mathrm{HS}}$ to a value of about 100 . Note that this effect cannot directly be seen in the left panel of figure 4 . This is because the constraints are only checked once, so spectra that are excluded because of the Higgs mass requirement can also be excluded by other constraints.

The optimal value for the ratio of $M_{2}$ to $M_{3}$ at the GUT scale is around 3 (figure 5), similar to what was reported in e.g. refs. [36, 144, 145]. However, unlike ref. [145] observes, we see that the ratio of $M_{1}$ to $M_{3}$ is less constrained. Moreover, we do not see the ellipsoid structure that ref. [36] observed. Our result can be understood by inspection of eq. (2.8). For moderate values of $\tan \beta$, the biggest contribution to $\Delta_{\mathrm{BG}}^{\mathrm{HS}}$ comes from the sensitivity of $m_{H_{u}}$ on the input parameters. As can be seen in eq. (2.8), the dependence of $m_{H_{u}}$ on the unified gaugino mass $M_{1 / 2}$ is minimal for $M_{2}=f_{2} M_{3} \simeq 2.7 M_{3}$ for a GUT scale value of $10^{16} \mathrm{GeV}$, SUSY scale of $1 \mathrm{TeV}$ and a $\tan \beta$ of 10 . This explains why we find $M_{2} / M_{3} \simeq 3$ in our scan. The bino mass parameter $M_{1}$ has a very small RGE coefficient, hence we don't expect the ratio of $M_{1}$ to $M_{3}$ to influence the value for $\Delta_{\mathrm{BG}}^{\mathrm{HS}}$ by a big amount, which is indeed what is observed in the figure.

\footnotetext{
${ }^{4}$ This number is slightly higher than the one reported in ref. [36], which is most likely caused by the fact that we take an updated set of constraints into account.
} 

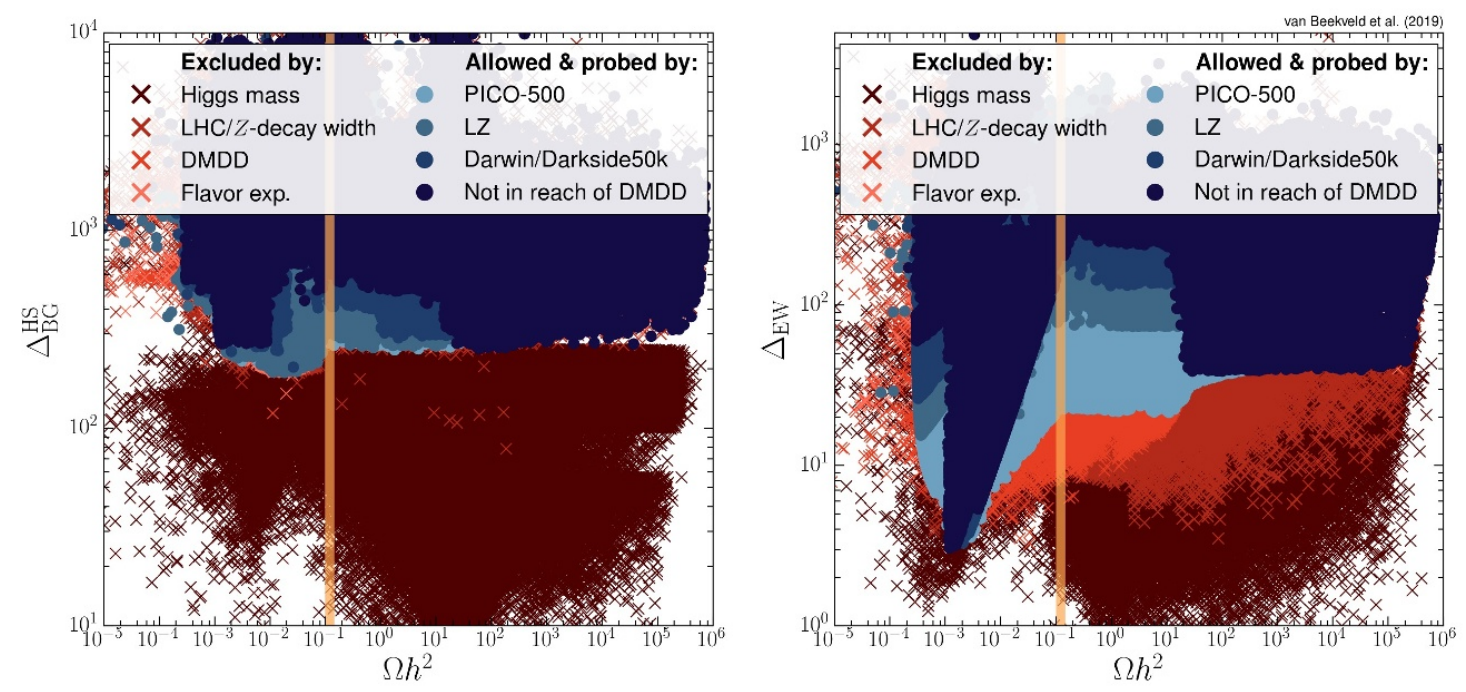

Figure 4. Generated spectra for the mSUGRA-var model. The left figure shows $\Delta_{\mathrm{BG}}^{\mathrm{HS}}$ as a function of $\Omega h^{2}$, the right figure shows $\Delta_{\mathrm{EW}}$ as a function of $\Omega h^{2}$. The color code and plotting order is the same as in figure 1. The orange band indicates $\Omega h^{2}=0.12$.

On the left-hand side of figure 6 it can be seen that $\Delta_{\mathrm{BG}}^{\mathrm{HS}}$ is again minimized in the region where $m_{\tilde{g}} \simeq 2 \mathrm{TeV}$ and $m_{\tilde{t}_{1}} \simeq 1 \mathrm{TeV}$, which is happening for the same reason as in the mSUGRA model. On the right-hand side of figure 6 , one can observe that the LSP can drop below $100 \mathrm{GeV}$. This can happen due to the increased freedom in the gaugino sector compared to the mSUGRA model. These low-mass LSPs are necessarily all binolike, otherwise $\tilde{\chi}_{1}^{ \pm}$would be excluded by LEP. One might wonder about the appearance of the two funnels around $m_{\tilde{\chi}_{1}^{0}} \simeq 45 \mathrm{GeV}$ and $m_{\tilde{\chi}_{1}^{0}} \simeq 65 \mathrm{GeV}$. All spectra surrounding these funnels are excluded by DMDD experiments, since for these spectra the higgsino or wino component is generally too high. However, in the funnel regions, these components are allowed to increase, as these spectra typically have values for $\Omega h^{2}$ that are less than 0.12 . This means that $m_{\tilde{\chi}_{1}^{ \pm}}$is allowed to be lower as well, which creates the funnels. The models that have $m_{\tilde{\chi}_{1}^{ \pm}} \simeq m_{\tilde{\chi}_{1}^{0}}$ have an LSP that is nearly $100 \%$ higgsino-like or wino-like. However, only $\tilde{\chi}_{1}^{ \pm}$and $\tilde{\chi}_{1}^{0}$ with small wino components feature in spectra that result in a low value of $\Delta_{\mathrm{BG}}^{\mathrm{HS}}$, which is due to the preferred GUT scale ratio of $M_{2} \simeq 2.7 M_{3}$. The spectra that minimize $\Delta_{\mathrm{BG}}^{\mathrm{HS}}$ all have a nearly-pure higgsino LSP with a mass around $200-500 \mathrm{GeV}$.

$\Delta_{\text {EW }}$ and the impact of DMDD experiments. The minimal value of $\Delta_{\mathrm{EW}}$ is 3 (right panel of figure 4), which is a factor of 10 smaller than in the mSUGRA model. The origin of this decrease is again caused by the increase in freedom of the gaugino sector. The radiative corrections of $m_{H_{u}}$ in both mSUGRA and mSUGRA-var are mainly driven by $M_{1 / 2}$ through $M_{3}$. Therefore, the value for $M_{1 / 2}$ at the GUT scale is constrained by the EWSB conditions. By no longer demanding the unifying condition of $M_{1}=M_{2}=M_{3}$ at the GUT-scale, one allows $M_{2}$ and $M_{1}$ to decouple from $M_{3}$. The decoupling creates a region in parameter space where the LSP is a pure higgsino LSP that is much larger than the region in mSUGRA. The purity of the LSP depletes the $\tilde{\chi}_{1}^{0} \tilde{\chi}_{1}^{0} Z$ coupling, therefore these 


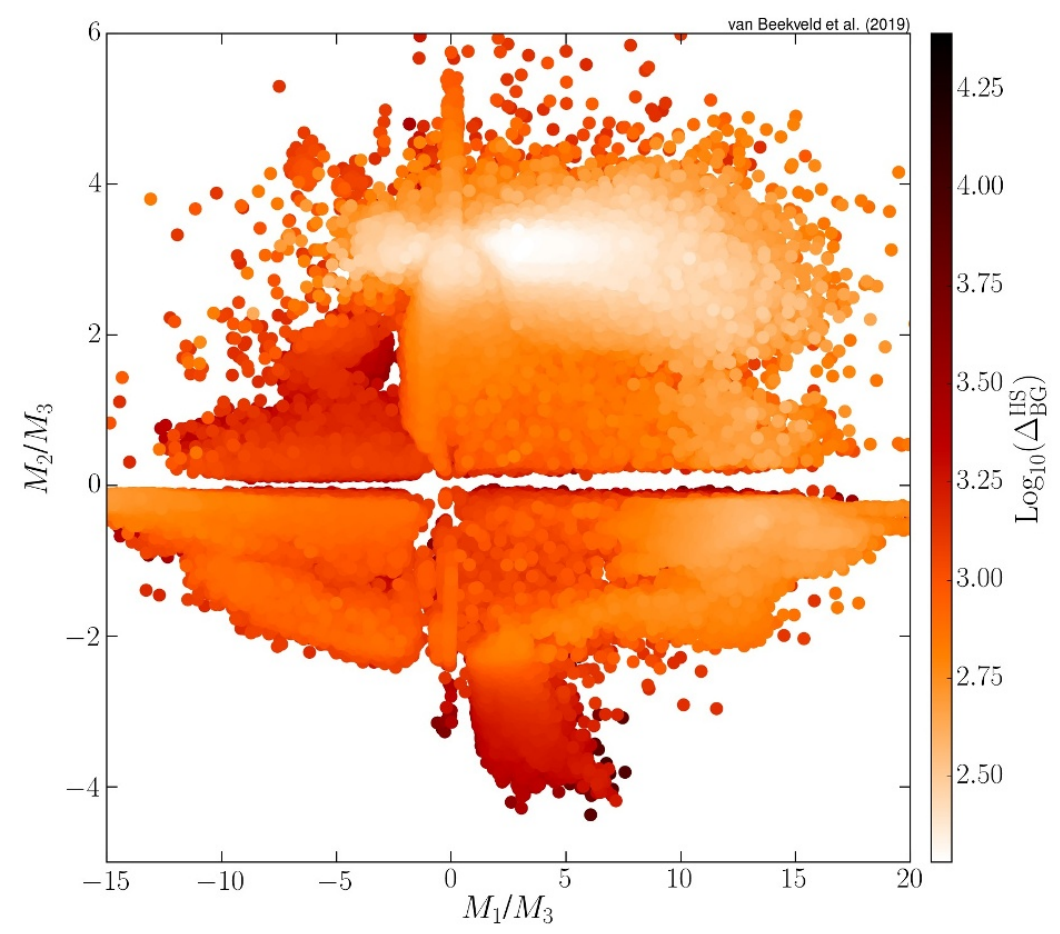

Figure 5. Ratios of $M_{1} / M_{3}$ and $M_{2} / M_{3}$ for the allowed mSUGRA-var spectra. The color code indicates the value for $\log _{10}\left(\Delta_{\mathrm{BG}}^{\mathrm{HS}}\right)$. Spectra with lower values for $\log _{10}\left(\Delta_{\mathrm{BG}}^{\mathrm{HS}}\right)$ lie on top of spectra with higher values.
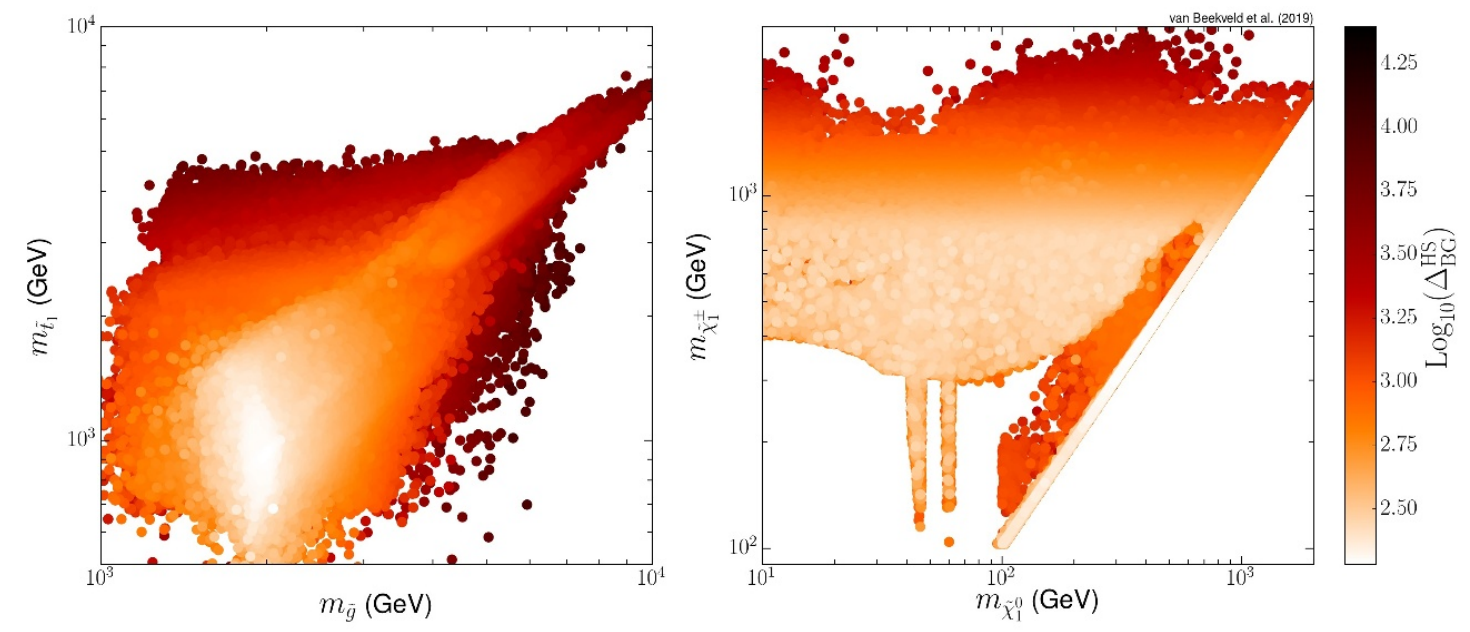

Figure 6. Left: $m_{\tilde{g}}$ against $m_{\tilde{t}_{1}}$ for mSUGRA-var, showing only the allowed spectra. Right: $m_{\tilde{\chi}_{1}^{ \pm}}$against $m_{\mathrm{DM}}$ for the allowed spectra. The masses are given in units of GeV. The color code indicates the value for $\log _{10}\left(\Delta_{\mathrm{BG}}^{\mathrm{HS}}\right)$. Spectra with lower values for $\log _{10}\left(\Delta_{\mathrm{BG}}^{\mathrm{HS}}\right)$ lie on top of spectra with higher values. 
spectra are not excluded by the DMDD experiments. Precisely these spectra minimize the value of $\Delta_{\mathrm{EW}}$.

Allowed spectra that result in a very low value for $\Omega h^{2}<10^{-3}$ have a wino-like LSP. These models were absent in mSUGRA since the wino mass parameter $M_{2}$ will never drop below $M_{1}$ at the SUSY scale due to the HS unification constraint. The LSPs of the allowed spectra with $\Omega h^{2} \simeq 10^{-4}$ and $\Delta_{\mathrm{EW}} \simeq 500$ have a mass around $100 \mathrm{GeV}$. The mass of the wino LSP increases for higher values of $\Omega h^{2}$. For slightly higher values of $10^{-3}<\Omega h^{2}<10^{-2}$, spectra appear that feature a pure higgsino LSP. The minimum of $\Delta_{\mathrm{EW}}$ is reached for spectra that have $\Omega h^{2} \simeq 10^{-3}$, corresponding to a pure higgsino LSP with a mass of $\mathcal{O}(100) \mathrm{GeV}$. As the LEP limits prevent $m_{\chi_{1}^{ \pm}}$to get smaller than $103.5 \mathrm{GeV}$, it is impossible to further minimize the value for $\Delta_{\mathrm{EW}}$. Future DMDD experiments are not able to constrain $\Delta_{\mathrm{EW}}$ in this regime. We do see a large sensitivity of the current and future DMDD experiments on $\Delta_{\mathrm{EW}}$ for spectra that saturate the dark matter relic density exactly. The minimal still allowed value of $\Delta_{\mathrm{EW}}$ for these spectra is 20 , while future DMDD experiments increase this value to 126. The spectra in this regime all have a bino-like LSP with a small higgsino component. The sensitivity of the future DMDD experiments decreases rapidly for $\Omega h^{2} \gtrsim 10$. This is explained due to the fact that in this regime spectra appear that have a bino-like LSP with a very small mass $(<10 \mathrm{GeV})$. In this mass regime, the DMDD experiments loose their sensitivity. To escape the limits on the size of the invisible $Z$-decay width, these light bino LSPs must have a negligible higgsino component. This prevents $|\mu|$ to get too low, which puts a lower limit on the value for $\Delta_{\mathrm{EW}}$ of around 40 . Note that also collider experiments constrain the wino component for these spectra. To escape detection at the LHC, a wino-like $\tilde{\chi}_{2}^{0}$ or $\tilde{\chi}_{1}^{ \pm}$needs to be heavier than about $600 \mathrm{GeV}[146,147]$. Future DMDD experiments constrain the minimal value of $\Delta_{\mathrm{BG}}^{\mathrm{HS}}$ to 252 . If we only consider the spectra that result in $0.09<\Omega h^{2}<0.15$, the future DMDD constrain $\Delta_{\mathrm{BG}}^{\mathrm{HS}}$ to 545 .

The impact of future collider experiments. The impact of the HL-LHC, HE-LHC and CLIC on the two FT measures can be seen in figure 7. Like in the mSUGRA model we observe that the impact on $\Delta_{\mathrm{BG}}^{\mathrm{HS}}$ is driven by the exclusion reach of $m_{\tilde{g}}$. The HL-LHC can increase the minimal value of $\Delta_{\mathrm{BG}}^{\mathrm{HS}}$ to about 530, and the HE-LHC can constrain it to about 1220. CLIC can constrain it to about 900 due to the sensitivity on $|\mu|$. The minimal value of $\Delta_{\mathrm{EW}}$ is not constrained by the exclusion reach of $m_{\tilde{g}}$, but only by the exclusion reach on $m_{\tilde{\chi}_{1}^{ \pm}}$. As the HL-LHC is able to probe $m_{\tilde{\chi}_{1}^{ \pm}}$up to about $350 \mathrm{GeV}, \Delta_{\mathrm{EW}}$ can be constrained to about 28 in the event of a non-observation, whereas the HE-LHC can constrain $\Delta_{\text {EW }}$ to about 70 . The exclusion reach of the HE-LHC on the stop mass increases the minimal value of $\Delta_{\mathrm{EW}}$ to about 60 . CLIC can constrain the value of $\Delta_{\mathrm{EW}}$ to about 530 .

\subsection{NUHGM}

In this HS model we don't assume a relation between $M_{1}, M_{2}$ and $M_{3}$, but instead treat them as free parameters. We furthermore increase the freedom of this model by having separate left and right-handed mass parameters for the sfermions and include $M_{A}$ and $\mu$ as 


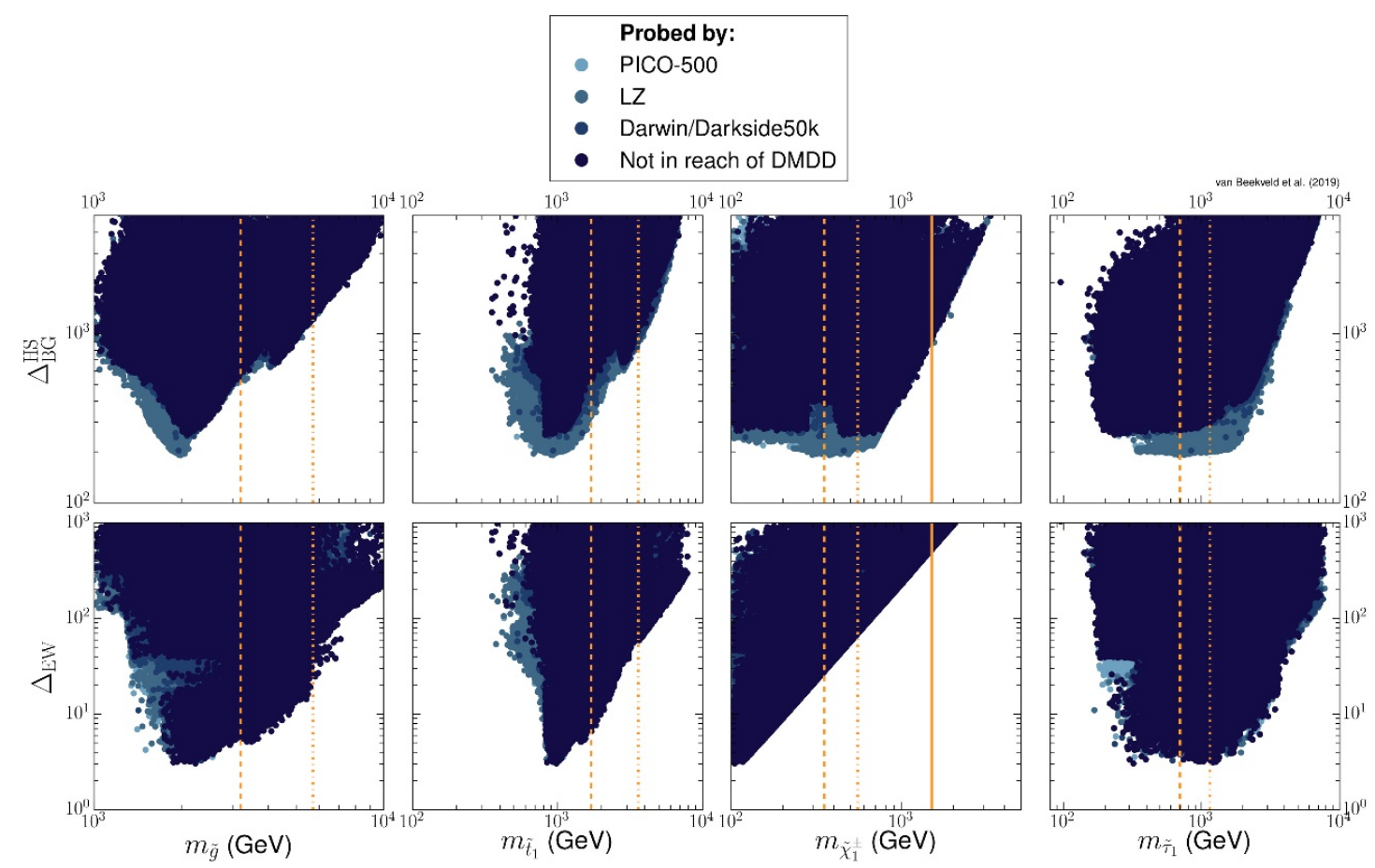

Figure 7. The values for $\Delta_{\mathrm{BG}}^{\mathrm{HS}}$ (top) and $\Delta_{\mathrm{EW}}$ (bottom) against (from left to right) $m_{\tilde{g}}, m_{\tilde{t}_{1}}$, $m_{\tilde{\chi}_{1}^{ \pm}}$and $m_{\tilde{\tau}_{1}}$ for the allowed mSUGRA-var spectra. The masses are given in units of GeV. The color code and plotting order is the same as in figure 1. The dashed, dash-dotted and solid orange line shows the exclusion potential of the HL-, HE-LHC and CLIC on the masses of various SUSY particles (see table 1). The solid orange line in the chargino mass plot shows the exclusion potential of CLIC.
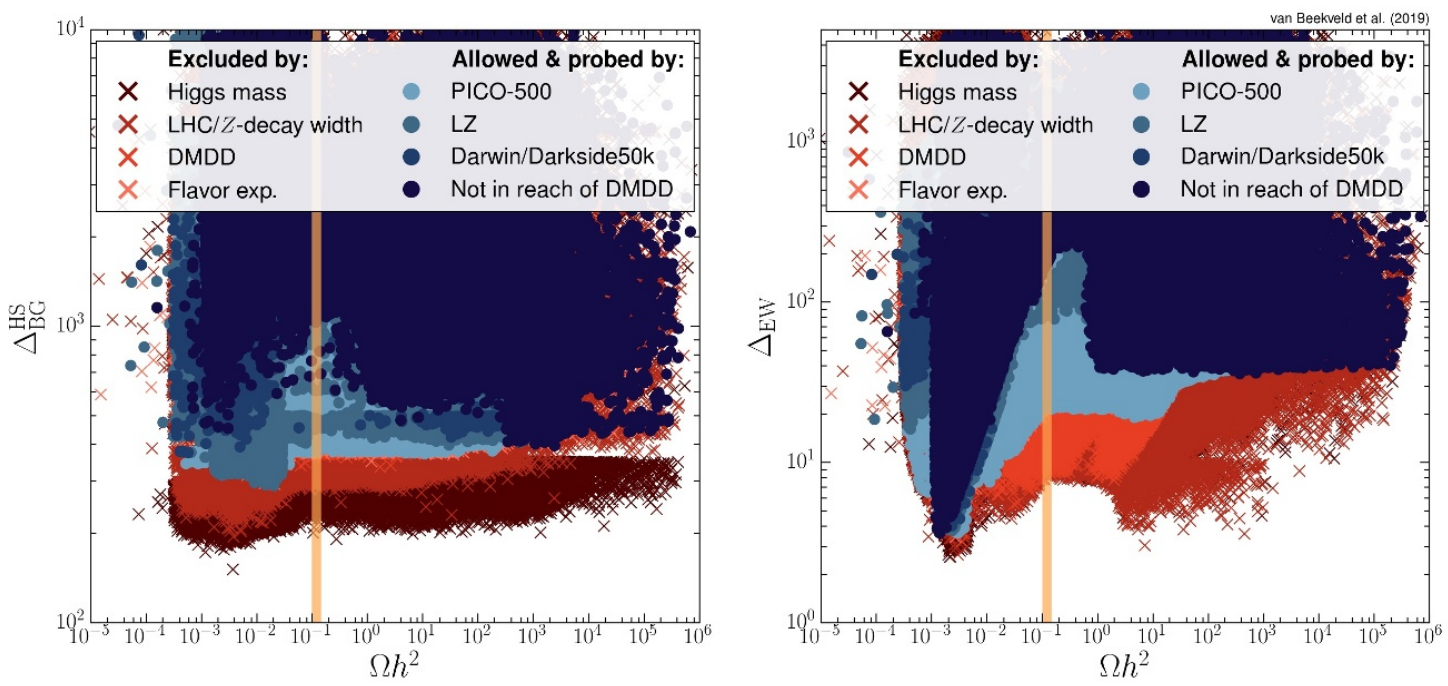

Figure 8. Generated spectra for the NUHGM model. The left figure shows $\Delta_{\mathrm{BG}}^{\mathrm{HS}}$ as a function of $\Omega h^{2}$, the right figure shows $\Delta_{\mathrm{EW}}$ as a function of $\Omega h^{2}$. The color code and plotting order is the same as in figure 1. The orange band indicates $\Omega h^{2}=0.12$. 


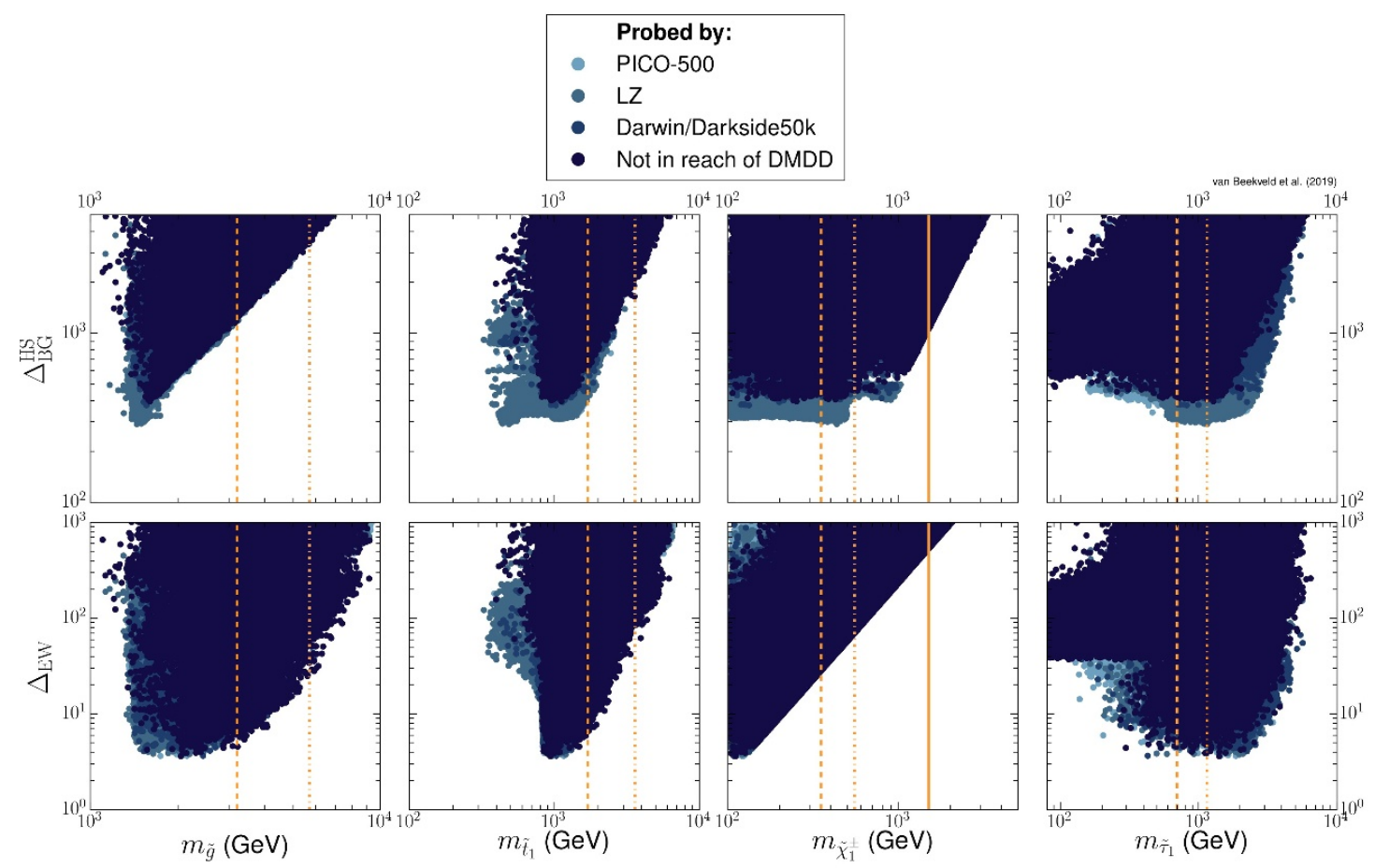

Figure 9. The values for $\Delta_{\mathrm{BG}}^{\mathrm{HS}}$ (top) and $\Delta_{\mathrm{EW}}$ (bottom) against (from left to right) $m_{\tilde{g}}, m_{\tilde{t}_{1}}, m_{\tilde{\chi}_{1}^{ \pm}}$ and $m_{\tilde{\tau}_{1}}$ for the allowed NUHGM spectra. The masses are given in units of GeV. The color code and plotting order is the same as in figure 1. The dashed, dash-dotted and solid orange line shows the exclusion potential of the HL-, HE-LHC and CLIC on the masses of various SUSY particles (see table 1). The solid orange line in the chargino mass plot shows the exclusion potential of CLIC.

input parameters. Although this model has more free parameters than the mSUGRA-var model, the resulting minimum for $\Delta_{\mathrm{BG}}^{\mathrm{HS}}$ is higher. The minimum value that we obtain for $\Delta_{\mathrm{BG}}^{\mathrm{HS}}$ is 290 . The increase with respect to the mSUGRA-var model, where the minimum of $\Delta_{\mathrm{BG}}^{\mathrm{HS}}$ was found at 191 , is due to the fact that now the ratios of $M_{1}$ and $M_{2}$ to $M_{3}$ are assumed to be independent at the HS. This indeed shows the dependence of $\Delta_{\mathrm{BG}}^{\mathrm{HS}}$ on the assumed HS model dependencies very clearly, as dropping the requirement of having a common parameter that generates mass for the entire gaugino sector increases the minimal value of $\Delta_{\mathrm{BG}}^{\mathrm{HS}}$ by 100 .

Different from the earlier discussed HS models, for NUHGM the minimal value for $\Delta_{\mathrm{BG}}^{\mathrm{HS}}$ is not only constrained by the Higgs mass requirement, but also by limits placed on SUSY particles. Dropping these two requirements decreases $\Delta_{\mathrm{BG}}^{\mathrm{HS}}$ with about 100. Another difference with respect to the previous two models is that now the minimal value for $\Delta_{\mathrm{BG}}^{\mathrm{HS}}$ is reached for the lowest still allowed value for $m_{\tilde{g}}$ and $m_{\tilde{t}_{1}}$ (figure 9). This is due to the fact that in this GUT model, $m_{0}$ and $M_{3}$ do not depend on $\mu$. This was not the case in the mSUGRA or mSUGRA-var model, where the value for $\mu$ is set by the value for $m_{0}$ and $M_{3}$ via the EWSB requirement.

$\Delta_{\text {EW }}$ and the impact of DMDD experiments. The minimal value obtained for $\Delta_{\mathrm{EW}}$ is again 3. As expected, the increase in freedom for the HS parameters in this 
model did not result in a lower value for $\Delta_{\mathrm{EW}}$. The same is true for spectra that result in $0.09<\Omega h^{2}<0.15$, where the minimal value for $\Delta_{\mathrm{EW}}$ is again found around 20. Future DMDD experiments are able to constrain $\Delta_{\mathrm{EW}}$ for these spectra to 146 . The spectra that escape detection by future DMDD experiments have pure higgsino LSPs with masses around $800 \mathrm{GeV}$. For values of $\Omega h^{2} \gtrsim 1$, the future DMDD experiments quickly lose their sensitivity, which is again caused by the presence of light $(\lesssim 10 \mathrm{GeV})$ bino-like LSPs. However, the value of $\Omega h^{2}$ where the DMDD experiments lose their constraining power is a factor of 10 lower than for the mSUGRA-var model. One can observe in the right panel of figure 9 that $m_{\tilde{\tau}_{1}}$ is allowed to drop below $\sim 200 \mathrm{GeV}$. In the mSUGRA-var model, this is not allowed, as then the Higgs mass requirement cannot be satisfied. In the present case, the small value for $m_{\tilde{\tau}_{1}}$ allows for a more efficient annihilation of LSPs into tau leptons via a t-channel $\tilde{\tau}_{1}$ exchange. This decreases the value of $\Omega h^{2}$, but at the same time does not give rise to a higher value for the SI or SD cross sections, as $\tilde{\tau}_{1}$ does not couple to nucleons directly.

The impact of future collider experiments. The impact of the HL-LHC, HE-LHC and CLIC on the two FT measures can be seen in figure 9. As before, we observe that the impact on $\Delta_{\mathrm{BG}}^{\mathrm{HS}}$ is driven by a higher reach on $m_{\tilde{g}}$. Surprisingly, the impact of the gluino mass exclusion on the minimal value of $\Delta_{\mathrm{BG}}^{\mathrm{HS}}$ is roughly a factor of 3-4 higher in this model than for mSUGRA-var. The HL-LHC can increase the minimal value of $\Delta_{\mathrm{BG}}^{\mathrm{HS}}$ to about 1195, while the HE-LHC constrains it about 3567. CLIC can constrain $\Delta_{\mathrm{BG}}^{\mathrm{HS}}$ to about 1070. The impact of the future colliders on the minimal value for $\Delta_{\mathrm{EW}}$ is similar here as to mSUGRA-var: the HL-LHC can increase $\Delta_{\mathrm{EW}}$ to about 28 , the HE-LHC can increase it to about 70, and CLIC can increase it to about 540, which is similar as in the mSUGRA-var model.

\section{4 pMSSM-GUT}

The last model we analyze is the pMSSM-GUT model. As explained in section 2.1, it has the same number of free parameters as the pMSSM model, but the input parameters are given at $M_{\mathrm{GUT}}$ (defined as the scale where the coupling constants $g_{1}, g_{2}$ and $g_{3}$ unify). Having essentially the same parameters at $M_{\mathrm{SUSY}}$ and at $M_{\mathrm{GUT}}$ allows us to study the influence of the RGE running on the obtained FT of a particular spectrum. To this end, we will use three different FT measures in this section. The first two are the same as before: the low scale FT measure $\Delta_{\mathrm{EW}}$ defined according to eq. (2.2) and the high scale FT measure $\Delta_{\mathrm{BG}}^{\mathrm{HS}}$ defined according to eq. (2.3). For the third FT measure we will use eq. (2.3), but set the matching conditions for the input parameters at $M_{\mathrm{SUSY}}=\sqrt{m_{\tilde{t}_{1}} m_{\tilde{t}_{2}}}$ instead of at $M_{\mathrm{GUT}}$, like in the case of the pMSSM. This FT measure will be indicated by $\Delta_{\mathrm{BG}}^{\mathrm{LS}}$. This section will be structured differently compared to the previous three sections, as here we will first compare the three FT measures, and subsequently move on to the discussion of some phenomenology of the spectra with the lowest FT values.

Comparison of the fine-tuning measures. We begin by comparing the two LS FT measures: $\Delta_{\mathrm{EW}}$ and $\Delta_{\mathrm{BG}}^{\mathrm{LS}}$, which are plotted against each other in the left panel of figure 10 . One observes that the two LS FT measures mostly agree for low values of $\Delta_{\mathrm{BG}}^{\mathrm{HS}}$, taken into 

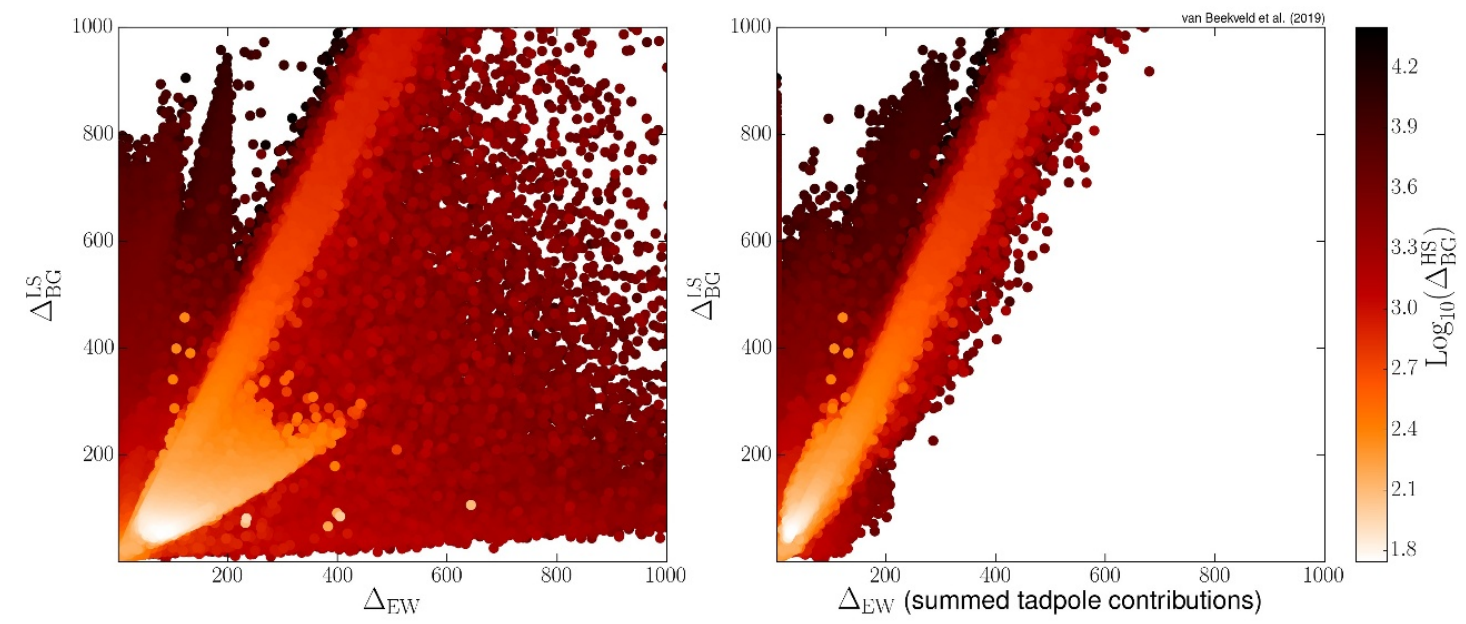

Figure 10. Left: $\Delta_{\mathrm{EW}}$ against $\Delta_{\mathrm{BG}}^{\mathrm{LS}}$ with $\log _{10}\left(\Delta_{\mathrm{BG}}^{\mathrm{HS}}\right)$ as color code. Here, $\Delta_{\mathrm{EW}}$ is computed considering each tadpole independently. Right: $\Delta_{\mathrm{EW}}$ against $\Delta_{\mathrm{BG}}^{\mathrm{LS}}$ where $\Delta_{\mathrm{EW}}$ is computed by summing up the tadpole contributions before evaluating their size. All computed pMSSM-GUT spectra are shown. Spectra with lower values for $\log _{10}\left(\Delta_{\mathrm{BG}}^{\mathrm{HS}}\right)$ lie on top of spectra with higher values.

account the fact that $\Delta_{\mathrm{BG}}^{\mathrm{LS}}$ is in general a factor of 2 higher than $\Delta_{\mathrm{EW}}$. This difference can indeed be traced back to the FT definitions in eq. (2.2) and eq. (2.3): $\mu$ is used as a fundamental parameter in eq. (2.3), which creates an extra factor of 2 as explained in section 2.3. The discrepancy between the measures generally grows for higher values of $\Delta_{\mathrm{BG}}^{\mathrm{HS}}$. We observe that $\Delta_{\mathrm{EW}}$ can either underestimate or overestimate $\Delta_{\mathrm{BG}}^{\mathrm{LS}}$. This overestimation happens because in the computation of $\Delta_{\mathrm{EW}}$, all tadpole contributions are assumed to be independent (see eq. (2.2)). When instead the total tadpole contributions are summed up, the spectra where $\Delta_{\mathrm{EW}}$ overestimates $\Delta_{\mathrm{BG}}^{\mathrm{LS}}$ mostly disappear, as can be seen on the right-hand side of figure 10. This phenomenon is observed for spectra for which the tadpole corrections belonging to the same particle type are large individually, but carry opposite signs. This may happen for example when the stop masses are degenerate (see appendix A of ref. [13]), and shows that it is not true that $\Delta_{\mathrm{EW}}$ is always the most conservative measure.

On the other hand, one can see that for some spectra the value for $\Delta_{\text {EW }}$ greatly underestimates the value for $\Delta_{\mathrm{BG}}^{\mathrm{LS}}$. The value for $\Delta_{\mathrm{EW}}$ for these spectra is determined by the size of $m_{H_{u}}$, while the value for $\Delta_{\mathrm{BG}}^{\mathrm{LS}}$ is mainly determined by variations in $m_{\tilde{Q}_{3}}, m_{\tilde{t}_{R}}$ and $M_{3}$. When the one-loop corrections of $m_{H_{u}}$ actually determine the size of $m_{H_{u}}$ (for example when the stop and/or gluino masses are large), the value of $\Delta_{\mathrm{BG}}^{\mathrm{LS}}$ will be driven by these parameters. In this case, when varying either one of these parameters, one induces a large change in $m_{H_{u}}$, which gives rise to a large value for $\Delta_{\mathrm{BG}}^{\mathrm{LS}}$. However, in $\Delta_{\mathrm{EW}}$, merely the size of $m_{H_{u}}$ and its tadpole terms are taken into account and these are not necessarily big for these spectra. It is precisely in this case that $\Delta_{\mathrm{EW}}$ can lead to an underestimation of $\Delta_{\mathrm{BG}}^{\mathrm{LS}}$. A second (subdominant) effect originates from the value of the SUSY scale. In general, a higher value for $M_{\mathrm{SUSY}}$ increases the dependence of $m_{H_{u}}$ on $M_{3}, m_{\tilde{Q}_{3}}$ and $m_{\tilde{t}_{R}}$, which can lead to $\Delta_{\mathrm{EW}}$ underestimating $\Delta_{\mathrm{BG}}^{\mathrm{LS}}$. 

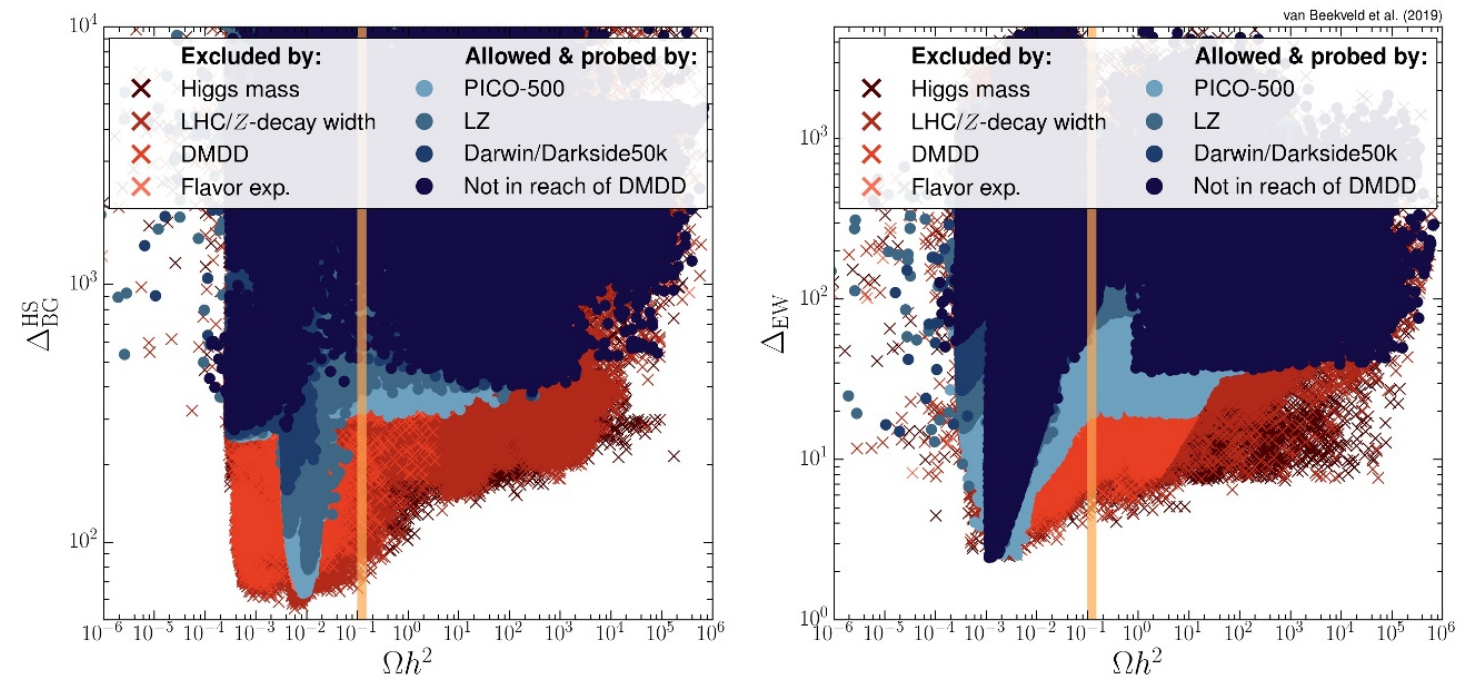

Figure 11. Generated spectra for the pMSSM-GUT model. The left figure shows $\Delta_{\mathrm{BG}}^{\mathrm{HS}}$ as a function of $\Omega h^{2}$, the right figure shows $\Delta_{\mathrm{EW}}$ as a function of $\Omega h^{2}$. The color code and plotting order is the same as in figure 1. The orange band indicates $\Omega h^{2}=0.12$.

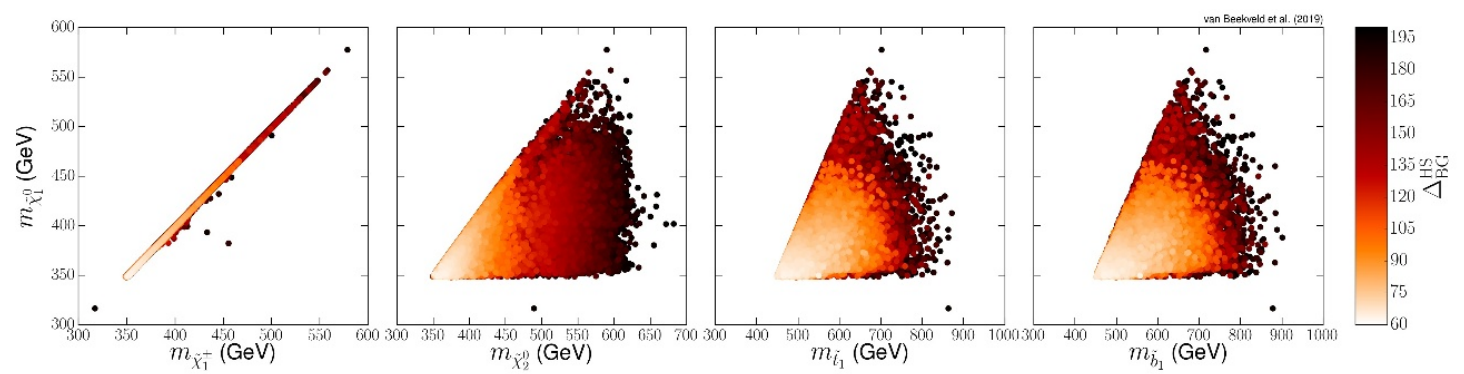

Figure 12. The masses of $\tilde{\chi}_{1}^{ \pm}, \tilde{\chi}_{2}^{0}, \tilde{t}_{1}$ and $\tilde{b}_{1}$ against $m_{\tilde{\chi}_{1}^{0}}$ in $\mathrm{GeV}$. The value for $\Delta_{\mathrm{BG}}^{\mathrm{HS}}$ is indicated by the color, and we only show spectra with $\Delta_{\mathrm{BG}}^{\mathrm{HS}}<200$. The spectra with lower values for $\Delta_{\mathrm{BG}}^{\mathrm{HS}}$ lie on top of those with higher values.

We now move on to the comparison between $\Delta_{\mathrm{BG}}^{\mathrm{HS}}$ and $\Delta_{\mathrm{BG}}^{\mathrm{LS}}$. In general, $\Delta_{\mathrm{BG}}^{\mathrm{HS}}$ will be larger than $\Delta_{\mathrm{BG}}^{\mathrm{LS}}$ due to the RGE running. Since the influence of the RGE running is almost absent in $\Delta_{\mathrm{BG}}^{\mathrm{LS}}$ for modest values $(\mathcal{O}(1) \mathrm{TeV})$ of the SUSY breaking scale, this leads to a reduced sensitivity of $m_{H_{u}}$ on e.g. $M_{3}, m_{\tilde{Q}_{3}}$ and $m_{\tilde{t}_{R}}$, and therefore a smaller value for $\Delta_{\mathrm{BG}}^{\mathrm{LS}}$ if these terms dominate its size. For $\Delta_{\mathrm{BG}}^{\mathrm{HS}}$ however, the effect of the RGE running cannot be neglected and in general the value of $\Delta_{\mathrm{BG}}^{\mathrm{HS}}$ will grow large for large values for the GUT values of $M_{3}, m_{\tilde{Q}_{3}}$ or $m_{\tilde{t}_{R}}$. Therefore, the discrepancy between $\Delta_{\mathrm{BG}}^{\mathrm{LS}}$ and $\Delta_{\mathrm{BG}}^{\mathrm{HS}}$ generally grows bigger for higher values of either $M_{3}, m_{\tilde{Q}_{3}}$ or $m_{\tilde{t}_{R}}$. Interestingly, we also find spectra where $\Delta_{\mathrm{BG}}^{\mathrm{LS}} \simeq \Delta_{\mathrm{BG}}^{\mathrm{HS}} \simeq 2 \Delta_{\mathrm{EW}}$. For these spectra, the value of $|\mu|$ determines the value of the FT for all measures. As $c_{\mu}$ in eq. (2.7) is close to 1 , for these spectra indeed only the size of $|\mu|$ matters in the computation of the FT and therefore the three FT measures reduce to approximately the same value. 

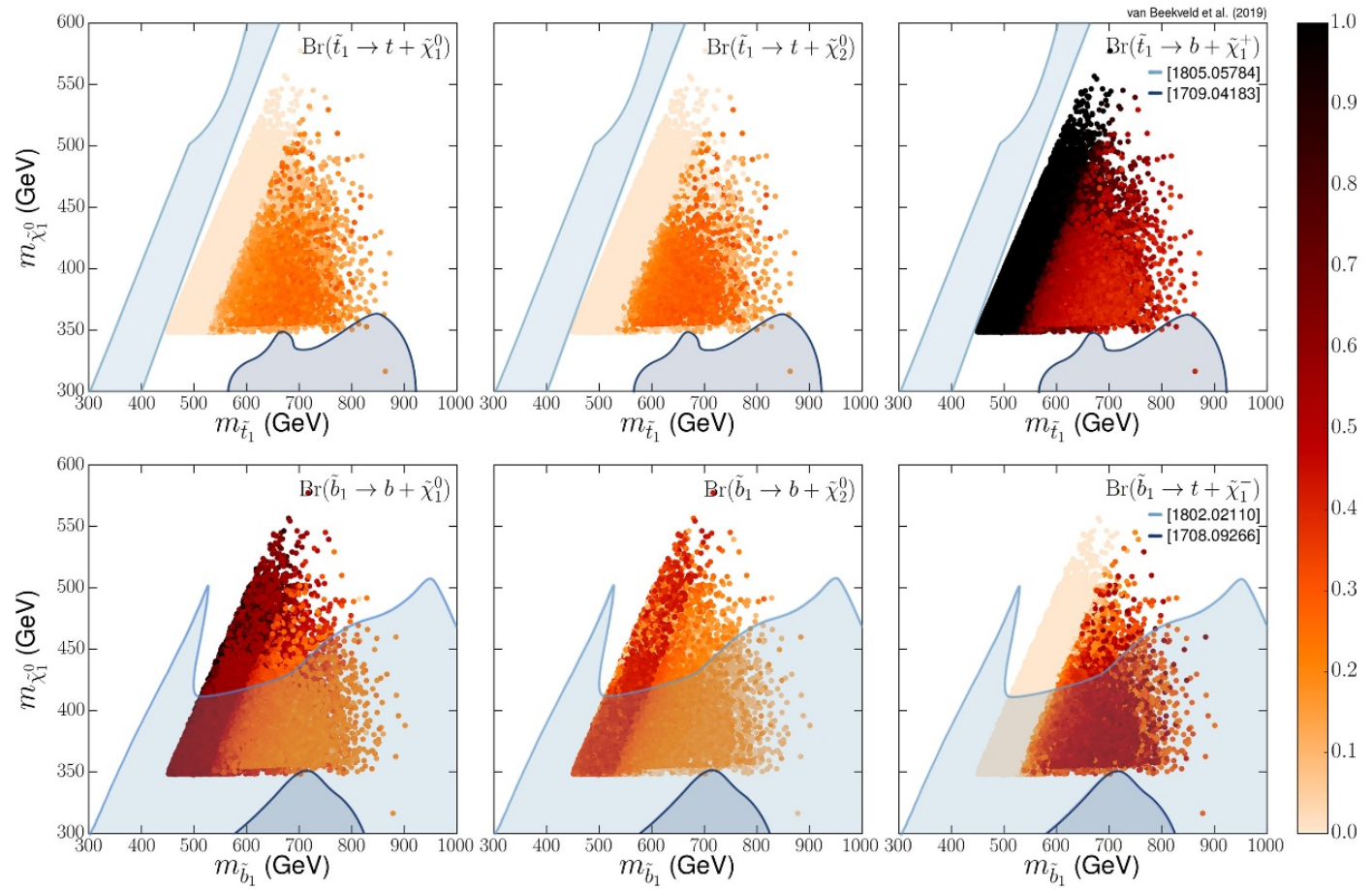

Figure 13. Branching ratios of $\tilde{t}_{1}$ and $\tilde{b}_{1}$ for the allowed pMSSM-GUT spectra with $\Delta_{\mathrm{BG}}<200$, where spectra with lower values for $\Delta_{\mathrm{BG}}^{\mathrm{HS}}$ lie on top of those with higher values. The top (bottom) row shows $m_{\tilde{t}_{1}}\left(m_{\tilde{b}_{1}}\right)$ against $m_{\tilde{\chi}_{1}^{0}}$ in $\mathrm{GeV}$, and the relevant decay process is indicated in the upper right corner of each plot. The two shaded areas in the plots on the top panel show the exclusion limits of ref. [148] (light blue), where $\operatorname{Br}\left(\tilde{t}_{1} \rightarrow t \tilde{\chi}_{1}^{0}\right)=100 \%$ is assumed, and ref. [149] (dark blue), where a mixed decay scenario is assumed. The two shaded areas in the plots of the bottom panel show the exclusion limits of ref. [150] (light blue), where $\operatorname{Br}\left(\tilde{b}_{1} \rightarrow b \tilde{\chi}_{1}^{0}\right)=100 \%$ is assumed, and ref. [151] (dark blue), where mixed decay scenarios are considered.

The phenomenology of low- $\Delta_{\mathrm{BG}}^{\mathrm{HS}}$ spectra. The resulting values for $\Delta_{\mathrm{BG}}^{\mathrm{HS}}$ (and $\Delta_{\mathrm{EW}}$ ) as a function of $\Omega h^{2}$ are shown in figure 11. The minimal value for $\Delta_{\mathrm{BG}}^{\mathrm{HS}}$ is 63 , which is lower than for all other GUT models previously considered. The spectra that minimize $\Delta_{\mathrm{BG}}^{\mathrm{HS}}$ all feature higgsino LSPs, and some of the LSPs also have a sizable wino component. Due to the presence of a higgsino asymmetry, and a non-zero wino component, the future DMDD experiments are sensitive to these spectra despite the fact that the relic density is not saturated. In figure 12 we show $m_{\tilde{\chi}_{1}^{0}}$ against $m_{\tilde{\chi}_{1}^{ \pm}}, m_{\tilde{\chi}_{2}^{0}}, m_{\tilde{t}_{1}}$ and $m_{\tilde{b}_{1}}$ of the allowed spectra with $\Delta_{\mathrm{BG}}^{\mathrm{HS}}<200$. These spectra are characterized by low values of $m_{\tilde{t}_{1}} \simeq 400-800 \mathrm{GeV}$ and $m_{\tilde{b}_{1}} \simeq 450-800 \mathrm{GeV}$. These masses are driven by $m_{\tilde{Q}_{3}}$, whose value lies around $100 \mathrm{GeV}$ at $M_{\mathrm{SUSY}}$. The masses of $\tilde{t}_{2}$ and $\tilde{b}_{2}$ are less constrained and have values ranging from $600 \mathrm{GeV}$ to $2 \mathrm{TeV}$. Surprisingly, the gluino mass is also unconstrained in this region. The lightest chargino is ultracompressed with the LSP, but their masses are too high to be discovered by the analysis of refs. [152, 153]. In the stop case, the analyses performed in refs. $[148,149,154]$ are most relevant when $\Delta\left(m_{\tilde{\chi}_{1}^{0}}, m_{\tilde{t}_{1}}\right)<m_{t}$. In this region, $\tilde{t}_{1}$ decays to $\tilde{\chi}_{1}^{+}$in association with a bottom quark with a branching ratio (BR) of $100 \%$ (see top panel of figure 13). The lightest chargino subsequently decays into a fermion-anti-fermion pair 

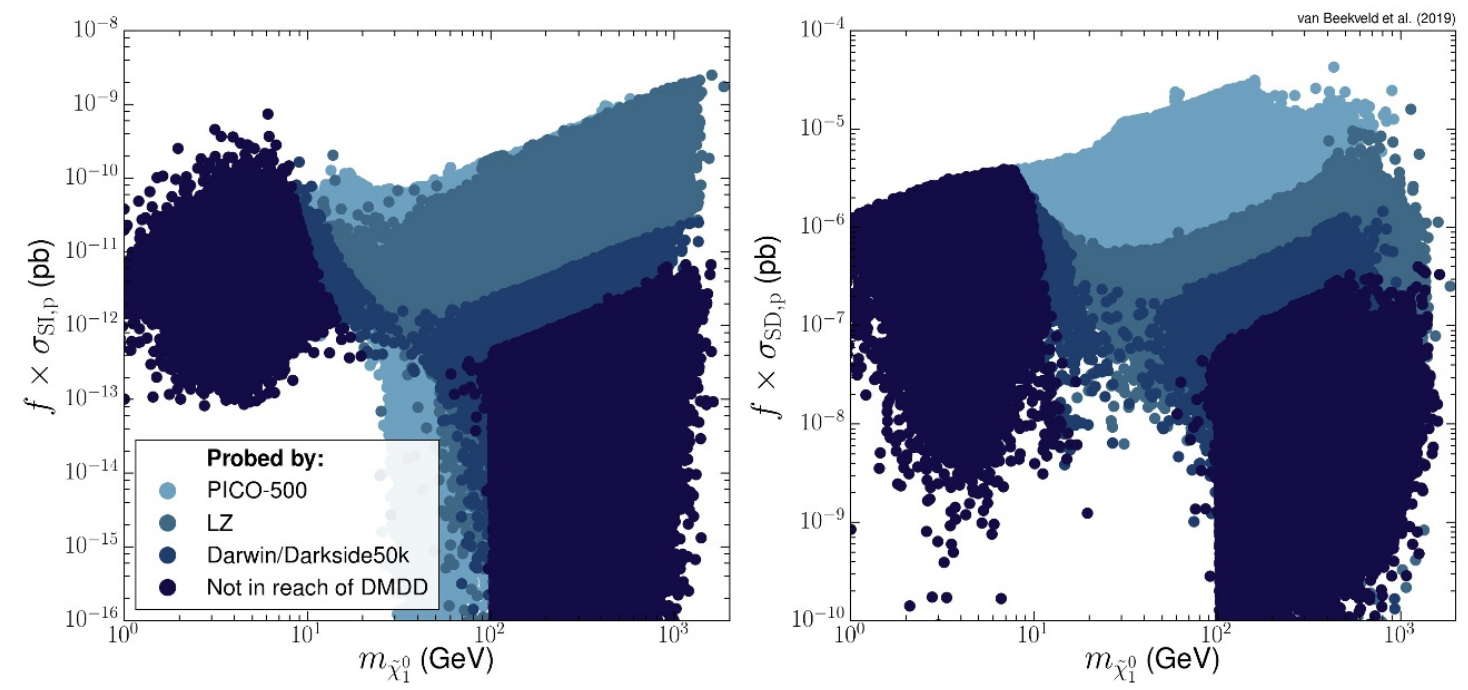

Figure 14. The mass of $\tilde{\chi}_{1}^{0}$ in GeV against $f \sigma_{\mathrm{SI}, \mathrm{p}}$ in pb (right) and $f \sigma_{\mathrm{SD}, \mathrm{p}}$ in pb (left), where $f$ is the rescaling factor when $\Omega h^{2}<0.12$. The color code and plotting order is the same as in figure 1 .

via an off-shell $W$-boson, where the fermions are ultra soft due to the mass compression between $\tilde{\chi}_{1}^{ \pm}$and $\tilde{\chi}_{1}^{0}$. One observes that the spectra where $\operatorname{Br}\left(\tilde{t}_{1} \rightarrow \tilde{\chi}_{1}^{+} b\right) \simeq 100 \%$ and $\Delta\left(m_{\tilde{\chi}_{1}^{0}}, m_{\tilde{t}_{1}}\right)<100 \mathrm{GeV}$ are under pressure, therefore we have introduced an explicit hard cut to exclude these spectra from our final results. In the case that $\Delta\left(m_{\tilde{\chi}_{1}^{0}}, m_{\tilde{t}_{1}}\right)>m_{t}$, the analyses in refs. $[149,154,155]$ are the most relevant, as we observe a mixed decay scenario for this region where $\operatorname{Br}\left(\tilde{t}_{1} \rightarrow \tilde{\chi}_{1}^{0} t\right) \simeq 25 \%, \operatorname{Br}\left(\tilde{t}_{1} \rightarrow \tilde{\chi}_{2}^{0} t\right) \simeq 25 \%$ and $\operatorname{Br}\left(\tilde{t}_{1} \rightarrow \tilde{\chi}_{1}^{+} b\right) \simeq 50 \%$.

In the sbottom case (lower panel of figure 13), the searches presented in ref. [150], where $\operatorname{Br}\left(\tilde{b}_{1} \rightarrow \tilde{\chi}_{1}^{0}+b\right)=100 \%$ is assumed, and in particular in ref. [151] are relevant, where the latter analysis considers a mixed sbottom decay scenario. The spectra with $\operatorname{Br}\left(\tilde{b}_{1} \rightarrow \tilde{\chi}_{1}^{0}+b\right)=100 \%$ are under pressure, and we exclude the spectra within the exclusion limit of ref. [150] that have $\operatorname{Br}\left(\tilde{b}_{1} \rightarrow \tilde{\chi}_{1}^{0}+b\right)>75 \%$. The spectra with the lowest FT values escape the limits set by the experiments, showing that a dedicated search for low mass sbottom and/or stop sparticles assuming mixed decay scenarios is needed to cover this region.

$\Delta_{\text {EW }}$ and the impact of DMDD experiments. The minimal value for $\Delta_{\mathrm{EW}}$ is again around 3 (figure 11). The spectra that result in $\Omega h^{2} \simeq 0.12$ give a minimum value of $\Delta_{\mathrm{EW}} \simeq 20$. The PICO 2019 limit on $\sigma_{\mathrm{SD}, \mathrm{p}}[104]$ is particularly constraining the minimally fine-tuned spectra that have the correct relic density. The LSPs that feature in these spectra are bino-higgsino mixtures, and have masses around $M_{Z} / 2, m_{h} / 2$ or $100 \mathrm{GeV}$. The lightest charginos have masses ranging from 100 to $300 \mathrm{GeV}$ for these spectra. The spectra that escape detection by future DMDD experiments are pure higgsino models, just like was the case for mSUGRA-var and NUHGM. In figure 14 we show $m_{\tilde{\chi}_{1}^{0}}$ against $f \sigma_{\mathrm{SI}, \mathrm{p}}$ and $f \sigma_{\mathrm{SD}, \mathrm{p}}$, where $f$ is the rescaling factor defined in section 3 . One observes a sharp increase in the number of spectra that evade the limits of future DMDD experiments completely around $m_{\tilde{\chi}_{1}^{0}}=100 \mathrm{GeV}$. These are the models with a pure higgsino LSP that deplete the 


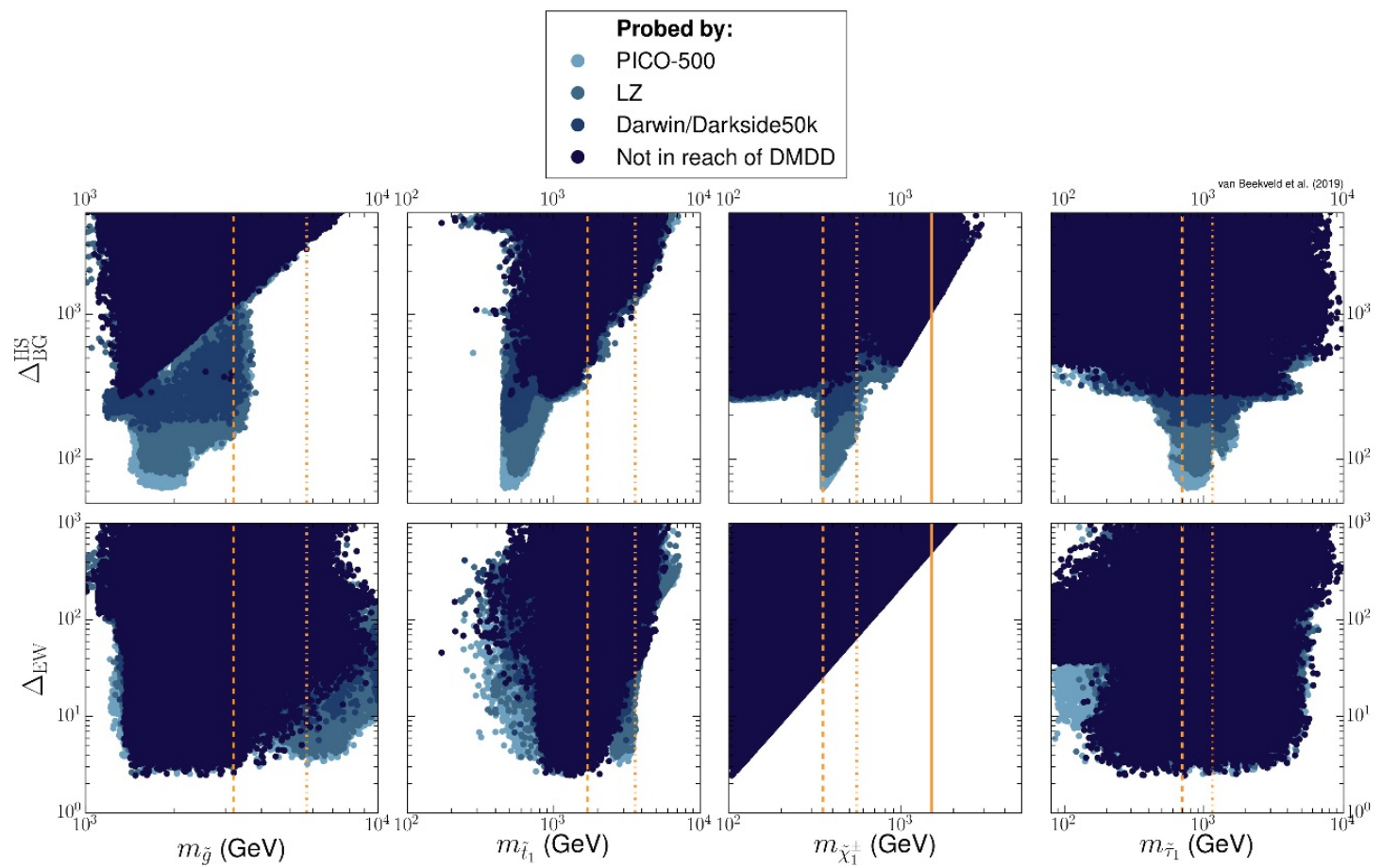

Figure 15. The values for $\Delta_{\mathrm{BG}}^{\mathrm{HS}}$ (top) and $\Delta_{\mathrm{EW}}$ (bottom) against (from left to right) $m_{\tilde{g}}, m_{\tilde{t}_{1}}$, $m_{\tilde{\chi}_{1}^{ \pm}}$and $m_{\tilde{\tau}_{1}}$ for all allowed pMSSM-GUT spectra. The masses are given in units of GeV. The color code and plotting order is the same as in figure 1. The dashed, dash-dotted and solid orange line shows the exclusion potential of the HL-, HE-LHC and CLIC on the masses of various SUSY particles (see table 1). The solid orange line in the chargino mass plot shows the exclusion potential of CLIC.

SD coupling due to the higgsino symmetry, and in addition have a rescaling factor of around $10^{-2}$. On the other hand, one observes that also LSPs with $m_{\tilde{\chi}_{1}^{0}} \lesssim 10 \mathrm{GeV}$ are out of reach of future DMDD experiments, as these simply are not sensitive to these mass ranges.

The impact of future collider experiments. The HL(HE)-LHC increases $\Delta_{\mathrm{EW}}$ to $\sim 70$ (28) by constraining the higgsino mass (figure 15), while CLIC constrains it to 540 . These numbers are similar to those that are found in the previous models. It was claimed before, that the requirement of having $\Delta_{\mathrm{EW}}<33$ places a limit on the gluino mass of around $6-9 \mathrm{TeV}[37,156,157]$, however, we do not observe that here. The HS FT measure $\Delta_{\mathrm{BG}}^{\mathrm{HS}}$ is again more constrained than $\Delta_{\mathrm{EW}}$ due to the increased gluino and stop mass reach of the HL- and HE-LHC. The HL-LHC can raise $\Delta_{\mathrm{BG}}^{\mathrm{HS}}$ to about 328 (driven by the limit that is placed on $m_{\tilde{t}_{1}}$ ), while the HE-LHC can raise $\Delta_{\mathrm{BG}}^{\mathrm{HS}}$ to about 3030 (due to the gluino mass reach). One observes a sharp increase in $\Delta_{\mathrm{BG}}^{\mathrm{HS}}$ for gluino masses around $3.5 \mathrm{TeV}$. There is no physical reason why this happens, it simply takes a large amount of computer resources to find these spectra. One can expect to find spectra with low values for $\Delta_{\mathrm{BG}}^{\mathrm{HS}}$ that have $m_{\tilde{g}} \gtrsim 3.5 \mathrm{TeV}$. CLIC can constrain the minimal value of $\Delta_{\mathrm{BG}}^{\mathrm{HS}}$ to 1100 .

Remarkable is that for all high-scale models, both FT measures result in roughly the same number in case of a non-observation at CLIC (taking into account the factor of 2 


\begin{tabular}{|c|c|c|c|c|c|}
\hline Model & Current & Future DD & HL-LHC & HE-LHC & CLIC \\
\hline mSUGRA $\Delta_{\mathrm{BG}}^{\mathrm{HS}}$ & $571(737)$ & $750(1032)$ & 848 & 1669 & 1237 \\
mSUGRA $\Delta_{\mathrm{EW}}$ & $38(110)$ & $275(515)$ & 39 & 86 & 529 \\
\hline mSUGRA-var $\Delta_{\mathrm{BG}}^{\mathrm{HS}}$ & $191(262)$ & $252(545)$ & 529 & 1222 & 888 \\
mSUGRA-var $\Delta_{\mathrm{EW}}$ & $3(20)$ & $3(126)$ & 28 & 70 & 529 \\
\hline NUHGM $\Delta_{\mathrm{BG}}^{\mathrm{HS}}$ & $290(375)$ & $395(691)$ & 1195 & 3567 & 1070 \\
NUHGM $\Delta_{\mathrm{EW}}$ & $3(20)$ & $3(146)$ & 28 & 70 & 537 \\
\hline pMSSM-GUT $\Delta_{\mathrm{BG}}^{\mathrm{HS}}$ & $63(272)$ & $328(517)$ & 372 & 3258 & 1108 \\
pMSSM-GUT $\Delta_{\mathrm{EW}}$ & $3(19)$ & $3(63)$ & 28 & 70 & 544 \\
\hline
\end{tabular}

Table 2. Summary of the high-scale models and their minimal amount of FT (rounded to integers). The first column indicates the high-scale model (definitions can be found in section 2.1). The second column shows the minimal FT both for $\Delta_{\mathrm{BG}}^{\mathrm{HS}}$ and $\Delta_{\mathrm{EW}}$ after applying only the current constraints. Between brackets we quote the result for the spectra that result in $0.09<\Omega h^{2}<0.15$. The third column gives the minimum of $\Delta_{\mathrm{BG}}^{\mathrm{HS}}$ and $\Delta_{\mathrm{EW}}$ after all the future DMDD experiments. The remaining columns give the maximal constraining power on $\Delta_{\mathrm{BG}}^{\mathrm{HS}}$ and $\Delta_{\mathrm{EW}}$ using the reach of the HL-LHC, HE-LHC and CLIC.

difference between $\Delta_{\mathrm{BG}}^{\mathrm{HS}}$ and $\left.\Delta_{\mathrm{EW}}\right)$. This shows that $|\mu|$ is the most model-independent parameter to determine the minimal possible amount of FT in the pMSSM.

One observes that $m_{\tilde{g}}$ is allowed to get as low as $1 \mathrm{TeV}$ for some spectra. These gluinos have a very complicated decay chain and they decay in at least three different neutralinos and one chargino. The mass differences between the neutralinos/charginos and the gluino are less than $50 \mathrm{GeV}$, and the chargino and heavier neutralino states decay into off-shell $W / Z$-bosons. Pair production of gluinos in the LHC thus mimics the QCD background, since the missing transverse energy (MET) is typically not large enough to discriminate the events. This allows them to escape detection at the LHC. Interestingly, these low mass gluinos do not appear in the spectra that create the minimum of $\Delta_{\mathrm{BG}}^{\mathrm{HS}}$ or $\Delta_{\mathrm{EW}}$. This is due to the fact that for both measures $|\mu|$ needs to be as low as possible. A low mass gluino is therefore excluded in spectra with low values for $\Delta_{\mathrm{BG}}^{\mathrm{HS}}$ or $\Delta_{\mathrm{EW}}$, as a low $|\mu|$-value reduces the mass compression of the NLSPs with the gluinos. This will allow pair production of the low mass gluinos to stand out of the QCD background due to a higher MET in these events.

\section{Conclusion}

In this paper, we have considered four different high-scale models, all with a low-scale realization in the pMSSM. We have minimized two different FT measures for these models, $\Delta_{\mathrm{EW}}$ (eq. 2.2) and $\Delta_{\mathrm{BG}}^{\mathrm{HS}}$ (eq. 2.3), and computed the impact of current/future collider experiments and current/future dark matter experiments on the minimal allowed FT. The results are summarized in table 2 and figure 16. In table 3, we contrast claims made in the literature on the status of FT in the pMSSM and possible GUT realizations with our findings. 

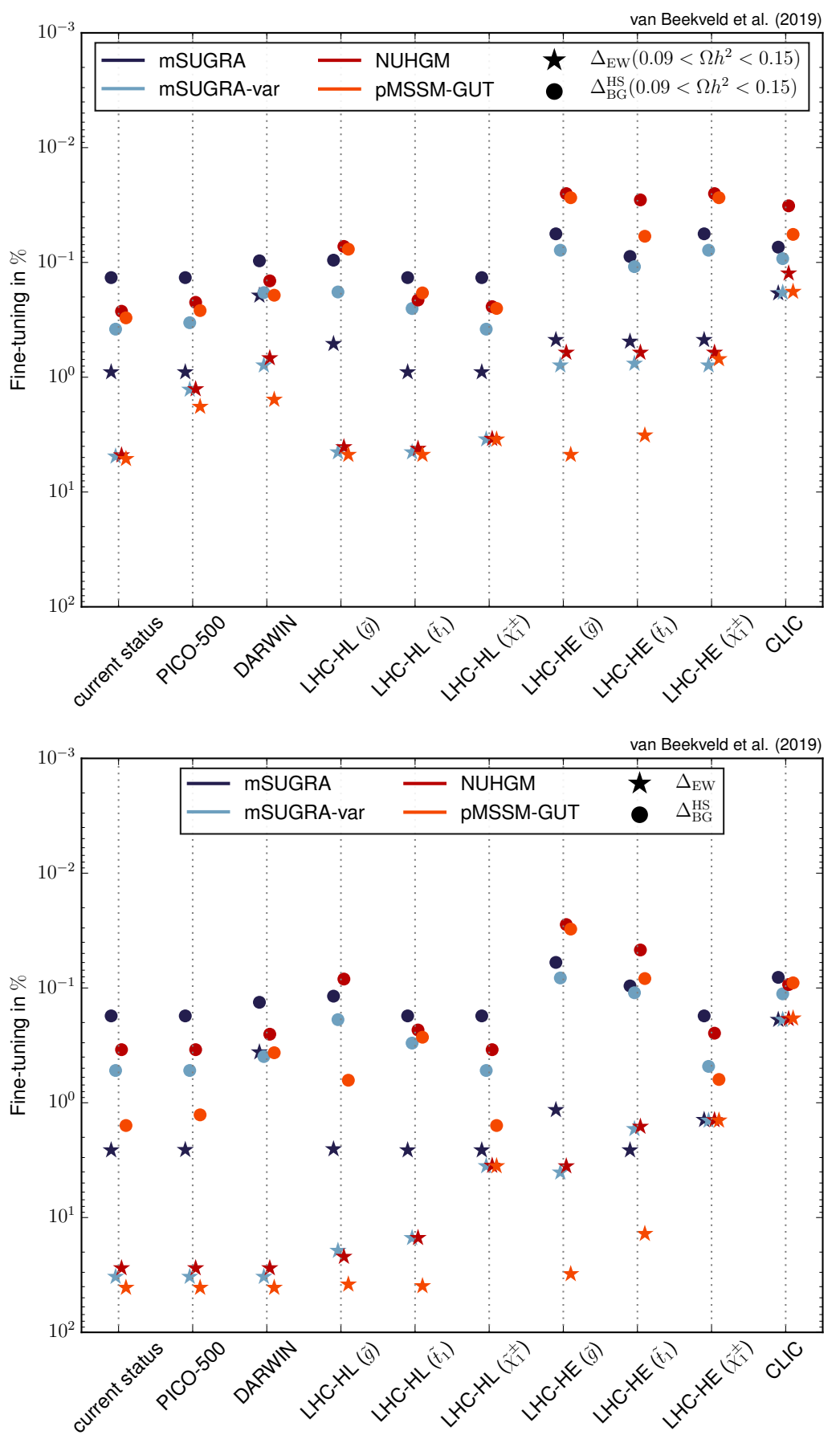

Figure 16. Summary of the high-scale models and minimal amount of FT still allowed after various future experiments with (top) and without (bottom) the requirement that $0.09<\Omega h^{2}<0.15$. The star (circle) shows the result for $\Delta_{\mathrm{EW}}\left(\Delta_{\mathrm{BG}}^{\mathrm{HS}}\right)$. The colors indicate various high-scale models, where dark blue indicates mSUGRA, light blue indicates mSUGRA-var, red indicates NUGHM and orange indicates pMSSM-GUT. 
We find that the obtained minimal FT values for $\Delta_{\mathrm{EW}}$ and $\Delta_{\mathrm{BG}}^{\mathrm{HS}}$ differ by at least an order of magnitude for each GUT model. For the mSUGRA and mSUGRA-var, the minimum of $\Delta_{\mathrm{BG}}^{\mathrm{HS}}$ is determined by the observed value for the Higgs boson mass, whereas for the NUHGM, the stop/gluino mass exclusion limits determine the minimum value of $\Delta_{\mathrm{BG}}^{\mathrm{HS}}$, whereas the stop and sbottom searches constrain the minimal value of $\Delta_{\mathrm{BG}}^{\mathrm{HS}}$ for the pMSSM-GUT model. We have found that the gluino searches are of lesser importance than is commonly assumed.

Interestingly, the minimal value of $\Delta_{\mathrm{BG}}^{\mathrm{HS}}$ is one order of magnitude lower in the pMSSMGUT model than for the other GUT models, whose minimal $\Delta_{\mathrm{BG}}^{\mathrm{HS}}$ values are of $\mathcal{O}(100)$. The minimum value for $\Delta_{\mathrm{BG}}^{\mathrm{HS}}$ in the pMSSM-GUT model is found at 63 , which corresponds to no more than $2 \%$ fine-tuning. This region could be further constrained by sbottom and stop searches at the LHC, where we stress that mixed decay scenarios should be further explored.

At the same time, the minimum of $\Delta_{\mathrm{EW}}$ is determined solely by $|\mu|$. The minimum value of this measure is less affected by the high-scale model. The minimal value that we have found for $\Delta_{\mathrm{EW}}$ is 3 (corresponding to $33 \%$ ) and is determined by the LEP chargino limits. The minimally fine-tuned spectra that predict the observed dark matter relic density are constrained by the dark matter direct detection experiments, and in particular by the PICO experiment [104]. The LSPs that feature in these spectra are bino-higgsino mixtures and have masses around $M_{Z} / 2, m_{h} / 2$ or $100 \mathrm{GeV}$. The lightest charginos have masses ranging from 100 to $300 \mathrm{GeV}$ for these spectra, which are unconstrained by the current LHC experiments as the production cross section is generally too small $[152,158]$.

As is known in the literature, the EW FT measure $\Delta_{\mathrm{EW}}$ can underestimate $\Delta_{\mathrm{BG}}^{\mathrm{LS}}$. However, in the pMSSM-GUT model, we have also seen that $\Delta_{\text {EW }}$ can also overestimate $\Delta_{\mathrm{BG}}^{\mathrm{LS}}$. This overestimation happens because in the computation of $\Delta_{\mathrm{EW}}$, all tadpole contributions are assumed to be independent (see eq. (2.2)). This phenomenon is observed for spectra for which the tadpole corrections belonging to the same particle type are large individually, but carry opposite signs. This may happen for example when the stop masses are degenerate (see appendix A of ref. [13]), and shows that it is not true that $\Delta_{\mathrm{EW}}$ is always the most conservative measure.

For all GUT models, we found spectra where $2 \Delta_{\mathrm{EW}}$ is roughly equal to $\Delta_{\mathrm{BG}}^{\mathrm{HS}}$. These spectra have in common that $|\mu|$ determines the value of $\Delta_{\mathrm{BG}}^{\mathrm{HS}}$. Therefore, to constrain the minimal FT in the most measure- and model-independent way, one must constrain $|\mu|{ }^{5}$ Future experiments that can constrain the value of $|\mu|$ are therefore favorable to settle the naturalness question of the pMSSM. Since a pure low-mass (around $100 \mathrm{GeV}$ ) higgsino LSP is produced under-abundantly in the early universe, the near-future DM direct detection experiments will not be sensitive to these spectra. Note that this statement changes if one considers only LSPs that satisfy the dark matter relic density constraint. In case of a non-observation, the future dark matter direct detection experiments can increase the limit on fine-tuning for spectra with LSPs that satisfy the dark matter relic density constraint from $19(5 \%)$ to $63(2 \%)$.

\footnotetext{
${ }^{5}$ There are ways to reduce the FT induced by $\mu$ that go beyond the MSSM (see ref. [159] for a recent overview), for example by including an explicit soft-SUSY breaking Higgsino mass term [36, 160].
} 


\begin{tabular}{|c|c|}
\hline Claim & This work \\
\hline $\begin{array}{l}\text { The pMSSM is not fine-tuned yet (e.g. [10, } \\
21,24,28,29,32,37,40,76,86])\end{array}$ & We support this claim. \\
\hline $\begin{array}{l}\text { The main requirement for low } \Delta_{\mathrm{EW}} \text { is that } \\
|\mu|=\mathcal{O}(100) \mathrm{GeV} \text { (e.g. }[10,21,24,28,29 \\
32,37,40,76,87]) \text {. }\end{array}$ & We support this claim. \\
\hline $\begin{array}{l}\text { Masses of pMSSM sparticles, and therefore } \\
\text { its parameters, need to be around } \mathcal{O}(0.1- \\
\text { 1) TeV, otherwise the pMSSM will be fine- } \\
\text { tuned (e.g. }[36,58-66,69,70,74]) \text {. }\end{array}$ & $\begin{array}{l}\text { We show that this statement is more nu- } \\
\text { anced: the }|\mu| \text { parameter certainly may not } \\
\text { grow too large, but all the other parameters } \\
\text { may be larger than } 1 \mathrm{TeV} \text {. In the pMSSM, } \\
\text { using } \Delta_{\mathrm{EW}} \text { to quantify the amount of FT, } \\
\text { we find a minimal value of } 3(33 \%) \text {, while } \\
\text { the masses of the colored sparticles are well } \\
\text { beyond } 1 \mathrm{TeV} \text {. }\end{array}$ \\
\hline $\begin{array}{l}\text { The gluino mass limits (and to a lesser ex- } \\
\text { tend, the stop mass limits) are the main } \\
\text { reason why the minimal value of } \Delta_{\mathrm{BG}}^{\mathrm{HS}} \text { is } \\
\text { constrained (e.g. }[17,27,35,38,51,53- \\
55,63,88] \text { ). }\end{array}$ & $\begin{array}{l}\text { We partially support this claim: it seems to } \\
\text { be true for the NUHGM scenario that the } \\
\text { gluino mass limits impact the minimal value } \\
\text { of } \Delta_{\mathrm{BG}}^{\mathrm{HS}} \text { directly. However, in the mSUGRA } \\
\text { scenarios, the Higgs mass requirement plays } \\
\text { a bigger role. In the pMSSM-GUT scenario, } \\
\text { the stop mass limit plays a bigger role. }\end{array}$ \\
\hline $\begin{array}{l}\Delta_{\mathrm{EW}} \text { will always be the conservative measure } \\
\text { (e.g. }[20,21,30,32,37,75,76,87])\end{array}$ & $\begin{array}{l}\text { In some cases, particularly when the stop } \\
\text { masses are degenerate, } \Delta_{\mathrm{BG}}^{\mathrm{HS}} \text { is lower than } \\
\Delta_{\mathrm{EW}} \text {. }\end{array}$ \\
\hline $\begin{array}{l}\text { The mSUGRA/CMSSM model has } \Delta_{\mathrm{BG}}^{\mathrm{HS}} \gtrsim \\
10^{3} \text { and } \Delta_{\mathrm{EW}} \gtrsim 10^{2}, \text { the NUHGM model has } \\
\Delta_{\mathrm{BG}}^{\mathrm{HS}} \gtrsim 10^{3} \text { and the pMSSM-GUT model has } \\
\left.\Delta_{\mathrm{BG}}^{\mathrm{HS}} \simeq 10 \text { (e.g. }[21,30,75]\right) .\end{array}$ & $\begin{array}{l}\text { mSUGRA can go as low as } \Delta_{\mathrm{BG}}^{\mathrm{HS}}=571 \\
\text { and } \Delta_{\mathrm{EW}}=38 \text {. The NUHGM model has } \\
\Delta_{\mathrm{BG}}^{\mathrm{HS}}>290 \text {, while the pMSSM-GUT model } \\
\text { has } \Delta_{\mathrm{BG}}^{\mathrm{HS}}=63 \text {. }\end{array}$ \\
\hline $\begin{array}{l}\text { Low } \Delta_{\mathrm{EW}} \text { spectra need } m_{\tilde{g}} \lesssim 5-7 \mathrm{TeV} \text { and } \\
m_{\tilde{t}_{1}} \lesssim 1-3 \mathrm{TeV}(\text { e.g. }[10,21,24,28-30,37 \\
37,156,157]) .\end{array}$ & $\begin{array}{l}\text { Low } \Delta_{\text {EW }} \text { spectra can easily have } m_{\tilde{g}}> \\
10 \mathrm{TeV} \text { and } m_{\tilde{t}_{1}} \text { up to at least } 3.5 \mathrm{TeV} .\end{array}$ \\
\hline
\end{tabular}

Table 3. Comparison of claims that are commonly made in the FT literature (after the discovery of the Higgs boson) with our findings.

On the other hand, dedicated higgsino searches at proton-proton colliders are particularly challenging due to small cross-sections (compared to QCD-induced processes or wino-induced production of the charginos and second-lightest neutralino) and low missing transverse energy (due to the mass compression). This makes the $|\mu|$ coverage relatively poor (see e.g. refs. [161, 162]). The ATLAS and CMS experiments both have targeted soft lepton searches to observe these particles, which seem to see small deviations from the SM background hypothesis in these regions [146, 163-165], although this deviation was not seen in the soft lepton analysis of ref. [166]. As can be seen in figure 16, the low- and high-scale fine-tuning measures reduce to the same value in case of a non-observation of higgsinos at CLIC. Therefore, CLIC may be the preferred collider to constrain the FT value in the most 
model-independent way. Our study shows that it is too early to conclude on the fate of the MSSM, and more measurements with less simplifying assumptions are needed to cover the parameter space of low fine-tuned MSSM spectra.

\section{Acknowledgments}

We wish to thank Wim Beenakker, Alberto Casas, Rohini Godbole and Ruud Peeters for useful discussions. R. RdA acknowledges partial funding/support from the Elusives ITN (Marie Skłodowska-Curie grant agreement No 674896), the "SOM Sabor y origen de la Materia" (FPA 2017-85985-P) and the Spanish MINECO Centro de Excelencia Severo Ochoa del IFIC program under grant SEV-2014-0398. MvB acknowledges support from the Dutch NWO-I program 156, "Higgs as Probe and Portal".

Open Access. This article is distributed under the terms of the Creative Commons Attribution License (CC-BY 4.0), which permits any use, distribution and reproduction in any medium, provided the original author(s) and source are credited.

\section{References}

[1] L. Susskind, Dynamics of spontaneous symmetry breaking in the Weinberg-Salam theory, Phys. Rev. D 20 (1979) 2619 [inSPIRE].

[2] M.J.G. Veltman, The infrared-ultraviolet connection, Acta Phys. Polon. B 12 (1981) 437 [INSPIRE].

[3] G. 't Hooft, Naturalness, chiral symmetry, and spontaneous chiral symmetry breaking, NATO Sci. Ser. B 59 (1980) 135.

[4] ATLAS collaboration, Observation of a new particle in the search for the Standard Model Higgs boson with the ATLAS detector at the LHC, Phys. Lett. B 716 (2012) 1 [arXiv: 1207.7214] [INSPIRE].

[5] CMS collaboration, Observation of a new boson at a mass of $125 \mathrm{GeV}$ with the CMS experiment at the LHC, Phys. Lett. B 716 (2012) 30 [arXiv:1207.7235] [INSPIRE].

[6] S.P. Martin, A supersymmetry primer, hep-ph/9709356.

[7] E. Witten, Dynamical breaking of supersymmetry, Nucl. Phys. B 188 (1981) 513.

[8] R.K. Kaul, Gauge hierarchy in a supersymmetric model, Phys. Lett. B 109 (1982) 19.

[9] N. Sakai, Naturalness in supersymmetric GUTs, Z. Phys. C 11 (1981) 153.

[10] H. Baer, V. Barger, P. Huang, A. Mustafayev and X. Tata, Radiative natural SUSY with a 125 GeV Higgs boson, Phys. Rev. Lett. 109 (2012) 161802 [arXiv:1207.3343] [INSPIRE].

[11] R. Kitano and Y. Nomura, Supersymmetry, naturalness, and signatures at the CERN LHC, Phys. Rev. D 73 (2006) 095004 [hep-ph/0602096].

[12] L.J. Hall, D. Pinner and J.T. Ruderman, A natural SUSY Higgs near 126 GeV, JHEP 04 (2012) 131 [arXiv: 1112.2703] [INSPIRE].

[13] H. Baer et al., Radiative natural supersymmetry: reconciling electroweak fine-tuning and the Higgs boson mass, Phys. Rev. D 87 (2013) 115028 [arXiv:1212.2655] [InSPIRE]. 
[14] R. Barbieri and G.F. Giudice, Upper bounds on supersymmetric particle masses, Nucl. Phys. B 306 (1988) 63 [inSPIRE].

[15] J.L. Feng, K.T. Matchev and T. Moroi, Focus points and naturalness in supersymmetry, Phys. Rev. D 61 (2000) 075005 [hep-ph/9909334] [InSPIRE].

[16] S. Antusch et al., Naturalness and GUT scale Yukawa coupling ratios in the CMSSM, Phys. Rev. D 85 (2012) 035025 [arXiv: 1111.6547] [InSPIRE].

[17] Z. Kang, J. Li and T. Li, On naturalness of the MSSM and NMSSM, JHEP 11 (2012) 024 [arXiv: 1201.5305] [INSPIRE].

[18] M.E. Cabrera, J.A. Casas and R. Ruiz de Austri, The health of SUSY after the Higgs discovery and the XENON100 data, JHEP 07 (2013) 182 [arXiv:1212.4821] [INSPIRE].

[19] S. Fichet, Quantified naturalness from Bayesian statistics, Phys. Rev. D 86 (2012) 125029 [arXiv:1204.4940] [INSPIRE].

[20] H. Baer et al., Post-LHC7 fine-tuning in the minimal supergravity/CMSSM model with a 125 GeV Higgs boson, Phys. Rev. D 87 (2013) 035017 [arXiv:1210.3019] [InSPIRE].

[21] H. Baer, V. Barger and M. Padeffke-Kirkland, Electroweak versus high scale finetuning in the 19-parameter SUGRA model, Phys. Rev. D 88 (2013) 055026 [arXiv:1304.6732] [INSPIRE].

[22] C. Balázs et al., Should we still believe in constrained supersymmetry?, Eur. Phys. J. C $\mathbf{7 3}$ (2013) 2563 [arXiv: 1205.1568] [INSPIRE].

[23] D. Kim et al., Bayesian naturalness of the CMSSM and CNMSSM, Phys. Rev. D 90 (2014) 055008 [arXiv: 1312.4150] [INSPIRE].

[24] H. Baer et al., Radiatively-driven natural supersymmetry at the LHC, JHEP 12 (2013) 013 [Erratum ibid. 06 (2015) 053] [arXiv:1310.4858] [INSPIRE].

[25] C. Boehm, P.S.B. Dev, A. Mazumdar and E. Pukartas, Naturalness of light neutralino dark matter in pMSSM after LHC, XENON100 and Planck data, JHEP 06 (2013) 113 [arXiv: 1303.5386] [INSPIRE].

[26] A. Fowlie, CMSSM, naturalness and the "fine-tuning price" of the Very Large Hadron Collider, Phys. Rev. D 90 (2014) 015010 [arXiv: 1403.3407] [InSPIRE].

[27] J.A. Casas et al., What is a Natural SUSY scenario?, JHEP 06 (2015) 070 [arXiv: 1407.6966] [INSPIRE].

[28] H. Baer et al., Natural SUSY with a bino- or wino-like LSP, Phys. Rev. D 91 (2015) 075005 [arXiv: 1501.06357] [INSPIRE].

[29] M. Drees and J.S. Kim, Minimal natural supersymmetry after the LHC8, Phys. Rev. D 93 (2016) 095005 [arXiv: 1511.04461] [inSPIRE].

[30] H. Baer, V. Barger and M. Savoy, Upper bounds on sparticle masses from naturalness or how to disprove weak scale supersymmetry, Phys. Rev. D 93 (2016) 035016 [arXiv: 1509.02929] [INSPIRE].

[31] J.A. Casas, J.M. Moreno, S. Robles and K. Rolbiecki, Reducing the fine-tuning of gauge-mediated SUSY breaking, Eur. Phys. J. C 76 (2016) 450 [arXiv:1602.06892] [INSPIRE].

[32] M. van Beekveld et al., Supersymmetry with Dark Matter is still natural, Phys. Rev. D 96 (2017) 035015 [arXiv: 1612.06333] [INSPIRE]. 
[33] A. Çiçi, Z. Kırca and C.S. Ün, Light stops and fine-tuning in MSSM, Eur. Phys. J. C 78 (2018) 60 [arXiv: 1611.05270] [InSPIRE].

[34] M.E. Cabrera et al., Naturalness of MSSM dark matter, JHEP 08 (2016) 058 [arXiv: 1604.02102] [INSPIRE].

[35] M.R. Buckley et al., Cornering natural SUSY at LHC Run II and beyond, JHEP 08 (2017) 115 [arXiv: 1610.08059$]$ [INSPIRE].

[36] G.G. Ross, K. Schmidt-Hoberg and F. Staub, Revisiting fine-tuning in the MSSM, JHEP 03 (2017) 021 [arXiv: 1701.03480] [INSPIRE].

[37] H. Baer et al., Reach of the high-energy LHC for gluinos and top squarks in SUSY models with light Higgsinos, Phys. Rev. D 96 (2017) 115008 [arXiv:1708.09054] [INSPIRE].

[38] M. Abdughani, L. Wu and J.M. Yang, Status and prospects of light bino-Higgsino dark matter in natural SUSY, Eur. Phys. J. C 78 (2018) 4 [arXiv:1705.09164] [InSPIRE].

[39] P. Fundira and A. Purves, Bayesian naturalness, simplicity and testability applied to the B-L MSSM GUT, Int. J. Mod. Phys. A 33 (2018) 1841004 [arXiv:1708.07835] [INSPIRE].

[40] H. Baer, V. Barger, D. Sengupta and X. Tata, Is natural higgsino-only dark matter excluded?, Eur. Phys. J. C 78 (2018) 838 [arXiv:1803.11210] [InSPIRE].

[41] F. Wang, K. Wang, J.M. Yang and J. Zhu, Solving the muon g- 2 anomaly in CMSSM extension with non-universal gaugino masses, JHEP 12 (2018) 041 [arXiv:1808.10851] [INSPIRE].

[42] J.R. Ellis, K. Enqvist, D.V. Nanopoulos and F. Zwirner, Observables in low-energy superstring models, Mod. Phys. Lett. A 1 (1986) 57 [INSPIRE].

[43] G.L. Kane, C.F. Kolda, L. Roszkowski and J.D. Wells, Study of constrained minimal supersymmetry, Phys. Rev. D 49 (1994) 6173 [hep-ph/9312272] [INSPIRE].

[44] G.W. Anderson and D.J. Castano, Measures of fine tuning, Phys. Lett. B 347 (1995) 300 [hep-ph/9409419] [INSPIRE].

[45] G.W. Anderson and D.J. Castano, Naturalness and superpartner masses or when to give up on weak scale supersymmetry, Phys. Rev. D 52 (1995) 1693 [hep-ph/9412322] [INSPIRE].

[46] S. Dimopoulos and G.F. Giudice, Naturalness constraints in supersymmetric theories with nonuniversal soft terms, Phys. Lett. B 357 (1995) 573 [hep-ph/9507282] [INSPIRE].

[47] P.H. Chankowski, J.R. Ellis and S. Pokorski, The fine tuning price of LEP, Phys. Lett. B 423 (1998) 327 [hep-ph/9712234] [INSPIRE].

[48] P.H. Chankowski, J.R. Ellis, M. Olechowski and S. Pokorski, Haggling over the fine tuning price of LEP, Nucl. Phys. B 544 (1999) 39 [hep-ph/9808275] [INSPIRE].

[49] J.A. Casas, J.R. Espinosa and I. Hidalgo, The MSSM fine tuning problem: a way out, JHEP 01 (2004) 008 [hep-ph/0310137] [INSPIRE].

[50] R. Kitano and Y. Nomura, A solution to the supersymmetric fine-tuning problem within the MSSM, Phys. Lett. B 631 (2005) 58 [hep-ph/0509039] [INSPIRE].

[51] M. Papucci, J.T. Ruderman and A. Weiler, Natural SUSY endures, JHEP 09 (2012) 035 [arXiv:1110.6926] [INSPIRE]. 
[52] M. Liu and P. Nath, Higgs boson mass, proton decay, naturalness and constraints of the LHC and Planck data, Phys. Rev. D 87 (2013) 095012 [arXiv:1303.7472] [InSPIRE].

[53] K. Kowalska and E.M. Sessolo, Natural MSSM after the LHC 8 TeV run, Phys. Rev. D 88 (2013) 075001 [arXiv:1307.5790] [INSPIRE].

[54] A. Arvanitaki et al., The last vestiges of naturalness, JHEP 03 (2014) 022 [arXiv:1309.3568] [INSPIRE].

[55] J. Lykken and M. Spiropulu, Supersymmetry and the crisis in physics, Sci. Am. 310N5 (2014) 36.

[56] S. Cassel, D.M. Ghilencea and G.G. Ross, Fine tuning as an indication of physics beyond the MSSM, Nucl. Phys. B 825 (2010) 203 [arXiv:0903.1115] [INSPIRE].

[57] G.G. Ross and K. Schmidt-Hoberg, The fine-tuning of the generalised NMSSM, Nucl. Phys. B 862 (2012) 710 [arXiv:1108.1284] [INSPIRE].

[58] I. Gogoladze, F. Nasir and Q. Shafi, Non-universal gaugino masses and natural supersymmetry, Int. J. Mod. Phys. A 28 (2013) 1350046 [arXiv:1212.2593] [INSPIRE].

[59] I. Gogoladze, F. Nasir and Q. Shafi, $\mathrm{SO}(10)$ as a framework for natural supersymmetry, JHEP 11 (2013) 173 [arXiv:1306.5699] [InSPIRE].

[60] A. Kaminska, G.G. Ross and K. Schmidt-Hoberg, Non-universal gaugino masses and fine tuning implications for SUSY searches in the MSSM and the GNMSSM, JHEP 11 (2013) 209 [arXiv: 1308.4168] [INSPIRE].

[61] J. Cao et al., Natural NMSSM after LHC Run I and the Higgsino dominated dark matter scenario, JHEP 08 (2016) 037 [arXiv: 1606.04416] [INSPIRE].

[62] J. Cao et al., Strong constraints of LUX-2016 results on the natural NMSSM, JHEP 10 (2016) 136 [arXiv: 1609.00204] [INSPIRE].

[63] C. Li, B. Zhu and T. Li, Naturalness, dark matter and the muon anomalous magnetic moment in supersymmetric extensions of the standard model with a pseudo-Dirac gluino, Nucl. Phys. B 927 (2018) 255 [arXiv: 1704.05584] [INSPIRE].

[64] B. Zhu, F. Staub and R. Ding, Naturalness and a light Z', Phys. Rev. D 96 (2017) 035038 [arXiv: 1707.03101] [INSPIRE].

[65] P. Athron et al., Bayesian analysis and naturalness of (next-to-)minimal supersymmetric models, JHEP 10 (2017) 160 [arXiv:1709.07895] [INSPIRE].

[66] C. Alvarado, A. Delgado and A. Martin, Constraining the R-symmetric chargino NLSP at the LHC, Phys. Rev. D 97 (2018) 115044 [arXiv: 1803.00624] [INSPIRE].

[67] X.K. Du et al., NMSSM with generalized deflected mirage mediation, Eur. Phys. J. C 79 (2019) 397 [arXiv: 1804.07335] [InSPIRE].

[68] M. Badziak and K. Harigaya, Impact of an extra gauge interaction on naturalness of supersymmetry, JHEP 08 (2018) 080 [arXiv: 1806.07900] [INSPIRE].

[69] A. Kobakhidze and M. Talia, Supersymmetric naturalness beyond MSSM, JHEP 08 (2019) 105 [arXiv: 1806.08502 ] [INSPIRE].

[70] T.T. Yanagida and N. Yokozaki, Focus point gauge mediation without a severe fine-tuning, JHEP 10 (2018) 149 [arXiv:1809.00787] [INSPIRE]. 
[71] M. Badziak, N. Desai, C. Hugonie and R. Ziegler, Extended gauge mediation in the NMSSM with displaced LHC signals, Eur. Phys. J. C 79 (2019) 67 [arXiv:1810.05618] [INSPIRE].

[72] J. Cao et al., Current status of a natural NMSSM in light of LHC $13 \mathrm{TeV}$ data and XENON-1T results, Phys. Rev. D 99 (2019) 075020 [arXiv:1810.09143] [INSPIRE].

[73] K. Wang, F. Wang, J. Zhu and Q. Jie, The semi-constrained NMSSM in light of muon $g-2$, LHC and dark matter constraints, Chin. Phys. C 42 (2018) 103109 [arXiv: 1811.04435] [INSPIRE].

[74] L. Delle Rose et al., Naturalness and dark matter in the supersymmetric B $-L$ extension of the standard model, Phys. Rev. D 96 (2017) 055004 [arXiv: 1702.01808] [InSPIRE].

[75] H. Baer, V. Barger and D. Mickelson, How conventional measures overestimate electroweak fine-tuning in supersymmetric theory, Phys. Rev. D 88 (2013) 095013 [arXiv:1309.2984] [INSPIRE].

[76] H. Baer, V. Barger, D. Mickelson and M. Padeffke-Kirkland, SUSY models under siege: LHC constraints and electroweak fine-tuning, Phys. Rev. D 89 (2014) 115019 [arXiv: 1404.2277] [INSPIRE].

[77] M.E. Cabrera, J.A. Casas and R. Ruiz de Austri, Bayesian approach and Naturalness in MSSM analyses for the LHC, JHEP 03 (2009) 075 [arXiv:0812.0536] [INSPIRE].

[78] MSSM WORKING GROUP, The minimal supersymmetric standard model: group summary report, talk given at GDR (Groupement De Recherche) - Supersymetrie, April 15-17, Montpellier, France (1998) [hep-ph/9901246].

[79] S.R. Coleman and E.J. Weinberg, Radiative corrections as the origin of spontaneous symmetry breaking, Phys. Rev. D 7 (1973) 1888 [inSPIRE].

[80] A.H. Chamseddine, R.L. Arnowitt and P. Nath, Locally supersymmetric grand unification, Phys. Rev. Lett. 49 (1982) 970 [INSPIRE].

[81] R. Barbieri, S. Ferrara and C.A. Savoy, Gauge models with spontaneously broken local supersymmetry, Phys. Lett. 119B (1982) 343 [INSPIRE].

[82] N. Ohta, Grand unified theories based on local supersymmetry, Prog. Theor. Phys. 70 (1983) 542 [INSPIRE].

[83] L.J. Hall, J.D. Lykken and S. Weinberg, Supergravity as the messenger of supersymmetry breaking, Phys. Rev. D 27 (1983) 2359 [INSPIRE].

[84] M.E. Cabrera, A. Casas, R. Ruiz de Austri and G. Bertone, LHC and dark matter phenomenology of the NUGHM, JHEP 12 (2014) 114 [arXiv:1311.7152] [INSPIRE].

[85] M. Peiro and S. Robles, Low-mass neutralino dark matter in supergravity scenarios: phenomenology and naturalness, JCAP 05 (2017) 010 [arXiv:1612.00460] [INSPIRE].

[86] A. Delgado, M. Quirós and C. Wagner, General focus point in the MSSM, JHEP 04 (2014) 093 [arXiv: 1402.1735] [INSPIRE].

[87] A. Mustafayev and X. Tata, Supersymmetry, naturalness and light Higgsinos, Indian J. Phys. 88 (2014) 991 [arXiv:1404.1386] [INSPIRE].

[88] M.R. Buckley, A. Monteux and D. Shih, Precision corrections to fine tuning in SUSY, JHEP 06 (2017) 103 [arXiv:1611.05873] [InSPIRE].

[89] J.H. Kotecha and P.M. Djuric, Gaussian particle filtering, IEEE Trans. Sign. Proc. 51 (2003) 2592. 
[90] B.C. Allanach, SOFTSUSY: a program for calculating supersymmetric spectra, Comput. Phys. Commun. 143 (2002) 305 [hep-ph/0104145] [INSPIRE].

[91] H. Bahl and W. Hollik, Precise prediction for the light MSSM Higgs boson mass combining effective field theory and fixed-order calculations, Eur. Phys. J. C 76 (2016) 499 [arXiv: 1608.01880] [inSPIRE].

[92] T. Hahn et al., High-precision predictions for the light CP-even Higgs boson mass of the minimal supersymmetric standard model, Phys. Rev. Lett. 112 (2014) 141801 [arXiv:1312.4937] [INSPIRE].

[93] M. Frank et al., The Higgs boson masses and mixings of the complex MSSM in the Feynman-diagrammatic approach, JHEP 02 (2007) 047 [hep-ph/0611326] [INSPIRE].

[94] G. Degrassi et al., Towards high precision predictions for the MSSM Higgs sector, Eur. Phys. J. C 28 (2003) 133 [hep-ph/0212020] [INSPIRE].

[95] S. Heinemeyer, W. Hollik and G. Weiglein, FeynHiggs: a program for the calculation of the masses of the neutral CP even Higgs bosons in the MSSM, Comput. Phys. Commun. 124 (2000) 76 [hep-ph/9812320] [INSPIRE].

[96] A. Djouadi, M.M. Muhlleitner and M. Spira, Decays of supersymmetric particles: The Program SUSY-HIT (SUspect-SdecaY-HDECAY-InTerface), Acta Phys. Polon. B 38 (2007) 635 [hep-ph/0609292] [INSPIRE].

[97] D. Barducci et al., Collider limits on new physics within MicrOMEGAs_4.3, Comput. Phys. Commun. 222 (2018) 327 [arXiv: 1606.03834] [INSPIRE].

[98] G. Bélanger et al., MicrOMEGAs5.0 : Freeze-in, Comput. Phys. Commun. 231 (2018) 173 [arXiv: 1801.03509] [INSPIRE].

[99] The GAMBIT Dark Matter Workgroup collaboration, DarkBit: a GAMBIT module for computing dark matter observables and likelihoods, Eur. Phys. J. C 77 (2017) 831 [arXiv:1705.07920] [INSPIRE].

[100] XENON collaboration, Dark matter search results from a one ton-year exposure of XENON1T, Phys. Rev. Lett. 121 (2018) 111302 [arXiv:1805.12562] [INSPIRE].

[101] XENON collaboration, Constraining the spin-dependent WIMP-nucleon cross sections with XENON1T, Phys. Rev. Lett. 122 (2019) 141301 [arXiv:1902.03234] [INSPIRE].

[102] PICO collaboration, Dark matter search results from the PICO- $60 C_{3} F_{8}$ bubble chamber, Phys. Rev. Lett. 118 (2017) 251301 [arXiv:1702.07666] [INSPIRE].

[103] PICO collaboration, Improved dark matter search results from PICO-2L Run 2, Phys. Rev. D 93 (2016) 061101 [arXiv:1601.03729] [INSPIRE].

[104] PICO collaboration, Dark matter search results from the complete exposure of the PICO-60 $C_{3} F_{8}$ bubble chamber, Phys. Rev. D 100 (2019) 022001 [arXiv:1902.04031] [INSPIRE].

[105] PandaX-II collaboration, Dark matter results from first 98.7 days of data from the PandaX-II experiment, Phys. Rev. Lett. 117 (2016) 121303 [arXiv: 1607.07400] [INSPIRE].

[106] PandaX-II collaboration, PandaX-II constraints on spin-dependent WIMP-nucleon effective interactions, Phys. Lett. B 792 (2019) 193 [arXiv:1807.01936] [INSPIRE].

[107] FERMI-LAT collaboration, Searching for dark matter annihilation from Milky Way dwarf spheroidal galaxies with six years of Fermi Large Area Telescope data, Phys. Rev. Lett. 115 (2015) 231301 [arXiv:1503.02641] [InSPIRE]. 
[108] Planck collaboration, Planck 2018 results. VI. Cosmological parameters, arXiv: 1807.06209 [INSPIRE].

[109] S. Caron et al., The BSM-AI project: SUSY-AI-generalizing LHC limits on supersymmetry with machine learning, Eur. Phys. J. C 77 (2017) 257 [arXiv:1605.02797] [INSPIRE].

[110] A. Barr and J. Liu, Analysing parameter space correlations of recent 13 TeV gluino and squark searches in the pMSSM, Eur. Phys. J. C 77 (2017) 202 [arXiv:1608.05379] [INSPIRE].

[111] F. Ambrogi et al., SModelS v1.2: long-lived particles, combination of signal regions and other novelties, arXiv:1811.10624 [INSPIRE].

[112] J. Heisig, S. Kraml and A. Lessa, Constraining new physics with searches for long-lived particles: Implementation into SModelS, Phys. Lett. B 788 (2019) 87 [arXiv:1808.05229] [INSPIRE].

[113] J. Dutta, S. Kraml, A. Lessa and W. Waltenberger, SModelS extension with the CMS supersymmetry search results from Run 2, LHEP 1 (2018) 5 [arXiv: 1803.02204] [INSPIRE].

[114] F. Ambrogi et al., SModelS v1.1 user manual: Improving simplified model constraints with efficiency maps, Comput. Phys. Commun. 227 (2018) 72 [arXiv:1701.06586] [INSPIRE].

[115] S. Kraml et al., SModelS: a tool for interpreting simplified-model results from the LHC and its application to supersymmetry, Eur. Phys. J. C 74 (2014) 2868 [arXiv:1312.4175] [INSPIRE].

[116] P. Bechtle et al., Applying exclusion likelihoods from LHC searches to extended Higgs sectors, Eur. Phys. J. C 75 (2015) 421 [arXiv:1507.06706] [InSPIRE].

[117] P. Bechtle et al., HiggsBounds-4: improved tests of extended Higgs sectors against exclusion bounds from LEP, the Tevatron and the LHC, Eur. Phys. J. C 74 (2014) 2693 [arXiv:1311.0055] [INSPIRE].

[118] P. Bechtle et al., Recent developments in HiggsBounds and a preview of HiggsSignals, PoS (CHARGED2012) 024 [arXiv: 1301.2345] [INSPIRE].

[119] P. Bechtle et al., HiggsBounds 2.0.0: confronting neutral and charged Higgs sector predictions with exclusion bounds from LEP and the Tevatron, Comput. Phys. Commun. 182 (2011) 2605 [arXiv: 1102.1898] [InSPIRE].

[120] P. Bechtle et al., HiggsBounds: confronting arbitrary Higgs sectors with exclusion bounds from LEP and the Tevatron, Comput. Phys. Commun. 181 (2010) 138 [arXiv:0811.4169] [INSPIRE].

[121] O. Stål and T. Stefaniak, Constraining extended Higgs sectors with HiggsSignals, PoS (EPS-HEP2013) 314 [arXiv:1310.4039] [INSPIRE].

[122] P. Bechtle et al., Probing the Standard Model with Higgs signal rates from the Tevatron, the LHC and a future ILC, JHEP 11 (2014) 039 [arXiv: 1403.1582] [INSPIRE].

[123] P. Bechtle et al., HiggsSignals: confronting arbitrary Higgs sectors with measurements at the Tevatron and the LHC, Eur. Phys. J. C 74 (2014) 2711 [arXiv:1305.1933] [INSPIRE].

[124] J.E. Camargo-Molina, B. O'Leary, W. Porod and F. Staub, Vevacious: a tool for finding the global minima of one-loop effective potentials with many scalars, Eur. Phys. J. C 73 (2013) 2588 [arXiv: 1307.1477] [INSPIRE]. 
[125] T.L. Lee, T.Y. Li, and C.H. Tsai, Hom4ps-2.0: a software package for solving polynomial systems by the polyhedral homotopy continuation method, Computing 83 (2008) 109.

[126] C.L. Wainwright, CosmoTransitions: computing cosmological phase transition temperatures and bubble profiles with multiple fields, Comput. Phys. Commun. 183 (2012) 2006 [arXiv:1109.4189] [INSPIRE].

[127] LEP2 SUSY Working Group, ALEPH, DELPHI, L3 and OPAL experiments, http://lepsusy.web.cern.ch/lepsusy.

[128] M. Carena, A. de Gouvêa, A. Freitas and M. Schmitt, Invisible $Z$ boson decays at $e^{+} e^{-}$ colliders, Phys. Rev. D 68 (2003) 113007 [hep-ph/0308053] [INSPIRE].

[129] M. Davier, A. Hoecker, B. Malaescu and Z. Zhang, Reevaluation of the hadronic contributions to the muon $g-2$ and to $\alpha_{M Z}$, Eur. Phys. J. C 71 (2011) 1515 [Erratum ibid. C 72 (2012) 1874] [arXiv: 1010.4180] [INSPIRE].

[130] LHCb collaboration, Measurement of the $B_{s}^{0} \rightarrow \mu^{+} \mu^{-}$branching fraction and search for $B^{0} \rightarrow \mu^{+} \mu^{-}$decays at the LHCb experiment, Phys. Rev. Lett. 111 (2013) 101805 [arXiv: 1307.5024] [INSPIRE].

[131] M. Misiak et al., Updated NNLO QCD predictions for the weak radiative B-meson decays, Phys. Rev. Lett. 114 (2015) 221801 [arXiv:1503.01789] [INSPIRE].

[132] M. Czakon et al., The $\left(Q_{7}, Q_{1,2}\right)$ contribution to $\bar{B} \rightarrow X_{s} \gamma$ at $\mathcal{O}\left(\alpha_{\mathrm{s}}^{2}\right)$, JHEP 04 (2015) 168 [arXiv: 1503.01791] [INSPIRE].

[133] B. Kronenbitter et al., Measurement of the branching fraction of $B^{+} \rightarrow \tau^{+} \nu_{\tau}$ decays with the semileptonic tagging method, Phys. Rev. D 92 (2015) 051102 [arXiv:1503.05613].

[134] Belle collaboration, Measurement of $B\left(D_{s}^{+} \rightarrow \mu_{\nu}\right)$, Phys. Rev. Lett. 100 (2008) 241801 [arXiv:0709.1340] [INSPIRE].

[135] CLEO collaboration, Improved measurement of absolute branching fraction of $D_{s}^{+} \rightarrow \tau^{+} \nu_{\tau}$, Phys. Rev. D 79 (2009) 052002 [arXiv:0901.1147] [INSPIRE].

[136] C. Strege et al., Profile likelihood maps of a 15-dimensional MSSM, JHEP 09 (2014) 081 [arXiv: 1405.0622] [INSPIRE].

[137] X. Cid Vidal et al., Report from working group 3, CERN Yellow Rep. Monogr. 7 (2019) 585 [arXiv: 1812.07831] [INSPIRE].

[138] CLICDP, CLIC collaboration, The Compact Linear Collider (CLIC) - 2018 Summary Report, CERN Yellow Rep. Monogr. 1802 (2018) 1 [arXiv:1812.06018] [INSPIRE].

[139] LZ collaboration, LUX-ZEPLIN (LZ) conceptual design report, arXiv:1509.02910 [INSPIRE].

[140] DARWIN collaboration, DARWIN: towards the ultimate dark matter detector, JCAP 11 (2016) 017 [arXiv: 1606.07001] [inSPIRE].

[141] M. Schumann, L. Baudis, L. Bütikofer, A. Kish and M. Selvi, Dark matter sensitivity of multi-ton liquid xenon detectors, JCAP 10 (2015) 016 [arXiv: 1506.08309] [INSPIRE].

[142] C.E. Aalseth et al., DarkSide-20k: a 20 tonne two-phase LAr TPC for direct dark matter detection at LNGS, Eur. Phys. J. Plus 133 (2018) 131 [arXiv:1707.08145] [InSPIRE].

[143] S. Fallows, Toward a next-generation dark matter search with the PICO-40L bubble chamber, in the proceedings of the Topics in Astroparticle and Underground Physics (TAUP 2017), July 24-28, Sudbury, Canada (2017). 
[144] S.P. Martin, Compressed supersymmetry and natural neutralino dark matter from top squark-mediated annihilation to top quarks, Phys. Rev. D 75 (2007) 115005 [hep-ph/0703097] [INSPIRE].

[145] S. Antusch et al., Naturalness of the non-Universal MSSM in the light of the recent Higgs results, JHEP 01 (2013) 187 [arXiv: 1207.7236] [INSPIRE].

[146] ATLAS collaboration, Search for chargino-neutralino production using recursive jigsaw reconstruction in final states with two or three charged leptons in proton-proton collisions at $\sqrt{s}=13$ TeV with the ATLAS detector, Phys. Rev. D 98 (2018) 092012 [arXiv: 1806. 02293] [INSPIRE].

[147] CMS collaboration, Searches for pair production of charginos and top squarks in final states with two oppositely charged leptons in proton-proton collisions at $\sqrt{s}=13 \mathrm{TeV}$, JHEP 11 (2018) 079 [arXiv: 1807.07799] [inSPIRE].

[148] CMS collaboration, Search for top squarks decaying via four-body or chargino-mediated modes in single-lepton final states in proton-proton collisions at $\sqrt{s}=13 \mathrm{TeV}$, JHEP 09 (2018) 065 [arXiv: 1805.05784] [InSPIRE].

[149] ATLAS collaboration, Search for a scalar partner of the top quark in the jets plus missing transverse momentum final state at $\sqrt{s}=13$ TeV with the ATLAS detector, JHEP 12 (2017) 085 [arXiv: 1709.04183] [InSPIRE].

[150] CMS collaboration, Search for natural and split supersymmetry in proton-proton collisions at $\sqrt{s}=13 \mathrm{TeV}$ in final states with jets and missing transverse momentum, JHEP 05 (2018) 025 [arXiv: 1802.02110] [INSPIRE].

[151] ATLAS collaboration, Search for supersymmetry in events with b-tagged jets and missing transverse momentum in pp collisions at $\sqrt{s}=13 \mathrm{TeV}$ with the ATLAS detector, JHEP 11 (2017) 195 [arXiv: 1708.09266] [INSPIRE].

[152] CMS collaboration, Search for new physics in events with two soft oppositely charged leptons and missing transverse momentum in proton-proton collisions at $\sqrt{s}=13 \mathrm{TeV}$, Phys. Lett. B 782 (2018) 440 [arXiv:1801.01846] [INSPIRE].

[153] ATLAS collaboration, Search for electroweak production of supersymmetric states in scenarios with compressed mass spectra at $\sqrt{s}=13 \mathrm{TeV}$ with the ATLAS detector, Phys. Rev. D 97 (2018) 052010 [arXiv:1712.08119] [InSPIRE].

[154] ATLAS collaboration, Search for top-squark pair production in final states with one lepton, jets and missing transverse momentum using $36 \mathrm{fb}^{-1}$ of $\sqrt{s}=13$ TeV pp collision data with the ATLAS detector, JHEP 06 (2018) 108 [arXiv:1711.11520] [INSPIRE].

[155] CMS collaboration, Search for top squarks and dark matter particles in opposite-charge dilepton final states at $\sqrt{s}=13$ TeV, Phys. Rev. D 97 (2018) 032009 [arXiv:1711.00752] [INSPIRE].

[156] H. Baer et al., What hadron collider is required to discover or falsify natural supersymmetry?, Phys. Lett. B 774 (2017) 451 [arXiv: 1702.06588] [INSPIRE].

[157] H. Baer et al., LHC luminosity and energy upgrades confront natural supersymmetry models, Phys. Rev. D 98 (2018) 075010 [arXiv: 1808.04844] [INSPIRE].

[158] M. van Beekveld, W. Beenakker, S. Caron and R. Ruiz de Austri, The case for $100 \mathrm{GeV}$ bino dark matter: A dedicated LHC tri-lepton search, JHEP 04 (2016) 154 [arXiv: 1602.00590] [INSPIRE]. 
[159] K.J. Bae, H. Baer, V. Barger and D. Sengupta, Revisiting the SUSY $\mu$ problem and its solutions in the LHC era, Phys. Rev. D 99 (2019) 115027 [arXiv:1902.10748] [INSPIRE].

[160] G.G. Ross, K. Schmidt-Hoberg and F. Staub, On the MSSM Higgsino mass and fine tuning, Phys. Lett. B 759 (2016) 110 [arXiv: 1603.09347] [INSPIRE].

[161] GAMBIT collaboration, A global fit of the MSSM with GAMBIT, Eur. Phys. J. C 77 (2017) 879 [arXiv: 1705.07917] [INSPIRE].

[162] GAMBIT collaboration, Combined collider constraints on neutralinos and charginos, Eur. Phys. J. C 79 (2019) 395 [arXiv:1809.02097] [INSPIRE].

[163] ATLAS collaboration, Search for electroweak production of supersymmetric particles in final states with two or three leptons at $\sqrt{s}=13 \mathrm{TeV}$ with the ATLAS detector, Eur. Phys. J. C 78 (2018) 995 [arXiv: 1803.02762] [InSPIRE].

[164] ATLAS collaboration, Search for supersymmetry in events with four or more leptons in $\sqrt{s}=13$ TeV pp collisions with ATLAS, Phys. Rev. D 98 (2018) 032009 [arXiv: 1804.03602] [INSPIRE].

[165] CMS collaboration, Search for new phenomena in final states with two opposite-charge, same-flavor leptons, jets and missing transverse momentum in pp collisions at $\sqrt{s}=13 \mathrm{TeV}$, JHEP 03 (2018) 076 [arXiv: 1709.08908] [INSPIRE].

[166] CMS collaboration, Combined search for electroweak production of charginos and neutralinos in proton-proton collisions at $\sqrt{s}=13 \mathrm{TeV}$, JHEP 03 (2018) 160 [arXiv: 1801.03957] [INSPIRE]. 